Research Article

\title{
The Fractional View Analysis of Polytropic Gas, Unsteady Flow System
}

\author{
Hassan Khan $(\mathbb{D})^{1,2}$ Saeed Islam $(\mathbb{D})^{3,4}$ and Muhammad Arif $\mathbb{D}^{1}$ \\ ${ }^{1}$ Department of Mathematics, Abdul Wali Khan University, Mardan 23200, Pakistan \\ ${ }^{2}$ Department of Mathematics, Near East University TRNC, Mersin 10, Turkey \\ ${ }^{3}$ Faculty of Mathematics \& Statistics, Ton Duc Thang University, Ho Chi Minh City 70000, Vietnam \\ ${ }^{4}$ Informatics Research Group, Ton Duc Thang University, Ho Chi Minh City 70000, Vietnam \\ Correspondence should be addressed to Saeed Islam; saeed.islam@tdtu.edu.vn
}

Received 20 August 2020; Revised 6 December 2020; Accepted 28 January 2021; Published 11 February 2021

Academic Editor: Lishan Liu

Copyright ( $) 2021$ Hassan Khan et al. This is an open access article distributed under the Creative Commons Attribution License, which permits unrestricted use, distribution, and reproduction in any medium, provided the original work is properly cited.

\begin{abstract}
Generally, the differential equations of integer order do not properly model various phenomena in different areas of science and engineering as compared to differential equations of fractional order. The fractional-order differential equations provide the useful dynamics of the physical system and thus provide the innovative and effective information about the given physical system. Keeping in view the above properties of fractional calculus, the present article is related to the analytical solution of the timefractional system of equations which describe the unsteady flow of polytropic gas dynamics. The present method provides the series form solution with easily computable components and a higher rate of convergence towards the targeted problem's exact solution. The present techniques are straightforward and effective for dealing with the solutions of fractional-order problems. The fractional derivatives are expressed in terms of the Caputo operator. The targeted problems' solutions are calculated using the Adomian decomposition method and variational iteration methods along with Shehu transformation. In the current procedures, we first applied the Shehu transform to reduce the problems into a more straightforward form and then implemented the decomposition and variational iteration methods to achieve the problems' comprehensive results. The solution of the nonlinear equations of unsteady flow of a polytropic gas at various fractional orders of the derivative is the core point of the present study. The solution of the proposed fractional model is plotted via two- and three-dimensional graphs. It is investigated that each problem's solution-graphs are best fitted with each other and with the exact solution. The convergence of fractional-order problems can be observed towards the solution of integer-order problems. Less computational time is the major attraction of the suggested methods. The present work will be considered a useful tool to handle the solution of fractional partial differential equations.
\end{abstract}

\section{Introduction}

In recent years, nonlinear fractional partial differential equations (FPDEs) have attracted researchers because of their useful applications in science and engineering [1-3]. The analysis of exact solutions to these nonlinear PDEs plays a very significant role in the Soliton theory since much of the information are provided on the description of the physical models, in the transmission of electrical signals, as a standard diffusion-wave equation, the transfer of neutrons by nuclear reactor, the theory of random walks, and so on [4-14].
In recent decades, many researchers have used different approaches to analyze the solutions of nonlinear PDEs, such as Laplace transform [15], Akbari-Ganji's method [16], homotopy analysis method [17], lattice Boltzmann method $[18,19]$, volume of fluid method [20, 21], Laplace homotopy analysis method [22, 23], Adomian decomposition technique [24-27], the variational iteration technique [28], Adams-Bashforth-Moulton algorithm [29], homotopy perturbation Sumudu transform method [30], the tanh method [31], the $\sin h$-cosh method [32], finite difference method [33], the homotopy perturbation method [34], and 
the Laplace decomposition technique, to handle fractionalorder Zakharov-Kuznetsov equations [35].

In the present study, we consider the gas-dynamic equations fractional-order scheme describing the evolution of an ideal gas's two-dimensional unsteady flow. The polytropic gas in astrophysics is given as follows [36]:

$$
\psi=k \omega^{1+(1 / m)}
$$

where $\psi=(\theta / \phi)$ is the energy density, $\phi$ is the container volume, $\theta$ is the total energy of the gas, $m$ is the polytropic index, and $k$ is a constant. Degenerate adiabatic gas and electron gas are two instances of such gases. In astrophysics and cosmology, the analysis of polytropic gases plays a critical role, and these gases can perform like dark energy [37]. Now consider the gas-dynamic equations scheme, which describes the evolution of unstable flow of a perfect gas with fractional derivatives $[36,38]$ :

$$
\begin{aligned}
& D_{\eta}^{\delta} \mu+\mu \frac{\partial \mu}{\partial \xi}+\nu \frac{\partial \mu}{\partial \zeta}+\frac{1}{\omega} \frac{\partial \psi}{\partial \xi}=0 \\
& D_{\eta}^{\delta} \nu+\mu \frac{\partial \nu}{\partial \xi}+\nu \frac{\partial \nu}{\partial \zeta}+\frac{1}{\omega} \frac{\partial \psi}{\partial \zeta}=0 \\
& D_{\eta}^{\delta} \omega+\mu \frac{\partial \omega}{\partial \xi}+\nu \frac{\partial \omega}{\partial \zeta}+\omega\left(\frac{\partial \mu}{\partial \xi}+\frac{\partial \nu}{\partial \zeta}\right)=0, \\
& D_{\eta}^{\delta} \psi+\mu \frac{\partial \psi}{\partial \xi}+\nu \frac{\partial \psi}{\partial \zeta}+\tau \psi\left(\frac{\partial \mu}{\partial \xi}+\frac{\partial \nu}{\partial \zeta}\right)=0,
\end{aligned}
$$

with initial conditions

$$
\begin{gathered}
\mu(\xi, \zeta, 0)=\alpha(\xi+\zeta), \\
\nu(\xi, \zeta, 0)=\beta(\xi+\zeta), \\
\omega(\xi, \zeta, 0)=\gamma(\xi+\zeta), \\
\psi(\xi, \zeta, 0)=\Phi(\xi+\zeta),
\end{gathered}
$$

where $\mu(\xi, \zeta, \eta)$ and $\nu(\xi, \zeta, \eta)$ are the velocity components, $\omega(\xi, \zeta, \eta)$ is the density, $\psi(\xi, \zeta, \eta)$ is the pressure, and $\tau$ is the ratio of the specific heat and it represents the adiabatic index. In past decade, the appropriate analytical results of several distinct types of gas-dynamic equations are achieved using many analytical and numerical methods. Various methods have been solved by a gas-dynamic model such as fractional reduced differential transform method [39], Elzaki transform homotopy perturbation method [40], $q$-homotopy analysis method [36], Adomian decomposition method [41], fractional homotopy analysis transform method [38], and natural decomposition method [42].

The variational iteration transform method (VITM) combines the variational iteration method and the Shehu transform. Many researchers commonly used this technique to solve linear and nonlinear models [43-45]. The method provides a reliable and effective procedure for a broad range of science. VIM does not need discretization, linearization, or perturbation. It provides quick convergence and successive approximations of the exact result [46-48]. Various equations solve the variational iteration method with the help of different transformations, such as Kuramoto-Sivashinsky equations [49] and fourth-order parabolic partial differential equation [45].

The ADM is an efficient and accurate technique that was suggested initially to solve analytically frontier physical models [50]. Since then, ADM has been implemented in nonlinear ODEs and PDEs without using perturbation or linearization procedure. The Shehu decomposition method (SDM) is a mixture of ADM and Shehu transform [51-54].

The motivation and novelty of the current research work are to modify the ADM and VIM along with Shehu transformation to investigate the solution of a nonlinear system of nonlinear FPDEs of unsteady flow of polytropic gas-dynamics equations. Besides the nonlinear system of four equations, the given problem's solution is calculated by an effortless and straightforward procedure. A higher degree of accuracy is achieved with a tiny number of calculations. The fractional-order solutions are achieved with some graphical justifications. The visual representation has confirmed the effectiveness and applicability of the suggested techniques. In the future, the proposed techniques are preferred to solve other nonlinear FPDEs that frequently arise in science and engineering.

\section{Preliminaries Concepts}

2.1. Definition 1. The Riemann-Liouville fractional integral is given as follows $[55,56]$ :

$$
\begin{aligned}
& I_{0}^{\delta} h(\eta)=\frac{1}{\Gamma(\delta)} \int_{0}^{\eta}(\eta-s)^{\delta-1} h(s) \mathrm{d} s, \quad \delta>0, \eta>0, \\
& h(\eta), \quad \delta
\end{aligned}
$$

2.2. Definition 2. The Caputo's fractional-order derivative of $h(\eta)$ is defined as follows $[55,56]$ :

$$
\begin{gathered}
D_{\eta}^{\delta} h(\eta)=I^{n-\delta} f^{n}, n-1<\delta<n, \quad n \in \mathbb{N} \\
\frac{d^{n}}{\mathrm{~d} \eta_{n}} h(\eta), \quad \delta=n, n \in \mathbb{N} .
\end{gathered}
$$

2.3. Definition 3. The Shehu transformation is the new and modern transformation which is described for exponentialorder functions. In set $A$, we take a function represented as follows [51, 52, 57, 58]:

$$
\begin{array}{r}
A=\left\{\nu(\eta): \exists, \rho_{1}, \rho_{2}>0,|\nu(\eta)|<\mathrm{Me}^{\left(|\eta| / \rho_{i}\right),},\right. \\
\text { if } \eta \in[0, \infty) .
\end{array}
$$

The Shehu transform which is given by $S($.$) for a$ function $v(\eta)$ is defined as

$$
\begin{gathered}
S\{\nu(\eta)\}=V(s, \mu)=\int_{0}^{\infty} e^{(-s \eta / \mu)} \nu(\eta) \mathrm{d} \eta, \\
\eta>0, s>0 .
\end{gathered}
$$


The Shehu transform of a function $\nu(\eta)$ is $V(s, \mu)$, and then, $v(\eta)$ is called the inverse of $V(s, \mu)$, which is given as

$$
\begin{aligned}
S^{-1}\{V(s, \mu)\}= & \nu(\eta), \text { for } \eta \geq 0, \\
& S^{-1} \text { is inverse Shehu transformation. }
\end{aligned}
$$

2.4. Definition 4. Shehu transform for $n$th derivatives is given as follows $[51,52,57,58]$ :

$$
S\left\{v^{(n)}(\eta)\right\}=\frac{s^{n}}{u^{n}} V(s, u)-\sum_{k=0}^{n-1}\left(\frac{s}{u}\right)^{n-k-1} v^{(k)}(0) .
$$

2.5. Definition 5. Shehu transform for fractional-order derivatives $[51,52,57,58]$ :

$$
\begin{array}{r}
S\left\{v^{(\delta)}(\eta)\right\}=\frac{s^{\delta}}{u^{\delta}} V(s, u)-\sum_{k=0}^{n-1}\left(\frac{s}{u}\right)^{\delta-k-1} v^{(k)}(0), \\
0<\beta \leq n .
\end{array}
$$

2.6. Definition 6. The Mittag-Leffler function denoted by $E_{\delta}(z)$ for $\delta>0$ is defined as

$$
E_{\delta}(z)=\sum_{m=0}^{\infty} \frac{z^{m}}{\Gamma(\delta m+1)} \delta>0, \quad z \in \mathbb{C} .
$$

\section{The Procedure of VITM}

This section describes the VITM solution, the system of FPDEs:

$$
\begin{array}{r}
D_{\eta}^{\delta} \mu(\xi, \zeta, \eta)+\overline{\mathscr{G}}_{1}(\mu, \nu)+\mathcal{N}_{1}(\mu, \nu)-\mathscr{G}_{1}(\xi, \zeta, \eta)=0, \\
D_{\eta}^{\delta} \nu(\xi, \zeta, \eta)+\overline{\mathscr{G}}_{2}(\mu, \nu)+\mathcal{N}_{2}(\mu, \nu)-\mathscr{G}_{2}(\xi, \zeta, \eta)=0, \\
m-1<\delta \leq m,
\end{array}
$$

with initial conditions

$$
\begin{aligned}
\mu(\xi, \zeta, 0) & =g_{1}(\xi, \zeta), \\
\nu(\xi, \zeta, 0) & =g_{2}(\xi, \zeta),
\end{aligned}
$$

where $D_{\eta}^{\delta}=\left(\partial^{\delta} / \partial \eta^{\delta}\right)$ is the Caputo fractional derivative of order $\delta ; \overline{\mathscr{G}}_{1}, \overline{\mathscr{G}}_{2}$ and $\mathcal{N}_{1}, \mathcal{N}_{2}$ are linear and nonlinear functions, respectively; and $\mathscr{G}_{1}, \mathscr{G}_{2}$ are source operators.

The Shehu transformation is applied to equation (1):

$$
\begin{aligned}
S & {\left[D_{\eta}^{\delta} \mu(\xi, \zeta, \eta)\right]+S\left[\overline{\mathscr{G}}_{1}(\mu, \nu)+\mathcal{N}_{1}(\mu, \nu)\right.} \\
& \left.-\mathscr{G}_{1}(\xi, \zeta, \eta)\right]=0, \\
& S\left[D_{\eta}^{\delta} \nu(\xi, \zeta, \eta)\right]+S\left[\overline{\mathscr{G}}_{2}(\mu, \nu)+\mathcal{N}_{2}(\mu, \nu)\right. \\
& \left.-\mathscr{G}_{2}(\xi, \zeta, \eta)\right]=0 .
\end{aligned}
$$

Applying the differentiation property of Shehu transform, we have

$$
\begin{aligned}
& S[\mu(\xi, \zeta, \eta)]-\left.\sum_{k=0}^{m-1} \frac{s^{\delta-k-1}}{u^{\delta-k}} \frac{\partial^{k} \mu(\xi, \zeta, \eta)}{\partial^{k} \eta}\right|_{\eta=0} \\
& =-S\left[\bar{G}_{1}(\mu, \nu)+\mathcal{N}_{1}(\mu, \nu)-\mathscr{G}_{1}(\xi, \zeta, \eta)\right] \\
& S[\nu(\xi, \zeta, \eta)]-\left.\sum_{k=0}^{m-1} \frac{s^{\delta-k-1}}{u^{\delta-k}} \frac{\partial^{k} \nu(\xi, \zeta, \eta)}{\partial^{k} \eta}\right|_{\eta=0} \\
& =-S\left[\overline{\mathscr{G}}_{2}(\mu, \nu)+\mathcal{N}_{2}(\mu, \nu)-\mathscr{G}_{2}(\xi, \zeta, \eta)\right] .
\end{aligned}
$$

The iteration method for equation (15) may be utilized to indicate the major iterative scheme requiring the Lagrange multiplier as

$$
\begin{aligned}
& S\left[\mu_{m+1}(\xi, \zeta, \eta)\right]=S\left[\mu_{m}(\xi, \zeta, \eta)\right]+ \\
& \lambda(s)\left[\frac{s^{\delta}}{u^{\delta}} \mu_{m}(\xi, \zeta, \eta)-\left.\sum_{k=0}^{m-1} \frac{s^{\delta-k-1}}{u^{\delta-k}} \frac{\partial^{k} \mu(\xi, \zeta, \eta)}{\partial^{k} \eta}\right|_{\eta=0}\right. \\
& \left.-S\left[\mathscr{G}_{1}(\xi, \zeta, \eta)\right]-S\left\{\overline{\mathscr{G}}_{1}(\mu, \nu)+\mathcal{N}_{1}(\mu, \nu)\right\}\right] \\
& S\left[\nu_{m+1}(\xi, \zeta, \eta)\right]=S\left[\nu_{m}(\xi, \zeta, \eta)\right]+\lambda(s)\left[\frac{s^{\delta}}{u^{\delta}}\right. \\
& \nu_{m}(\xi, \zeta, \eta)-\left.\sum_{k=0}^{m-1} \frac{s^{\delta-k-1}}{u^{\delta-k}} \frac{\partial^{k} \nu(\xi, \zeta, \eta)}{\partial^{k} \eta}\right|_{\eta=0} \\
& \left.-S\left[\mathscr{G}_{2}(\xi, \zeta, \eta)\right]-S\left\{\overline{\mathscr{G}}_{2}(\mu, \nu)+\mathcal{N}_{2}(\mu, \nu)\right\}\right]
\end{aligned}
$$

A Lagrange multiplier

$$
\lambda(s)=-\frac{u^{\delta}}{s^{\delta}}
$$

using inverse Shehu transformation $S^{-1}$, and equation (16) can be written as

$$
\begin{aligned}
\mu_{m+1}(\xi, \zeta, \eta) & \mu_{m}(\xi, \zeta, \eta)-S^{-1}\left[\frac { u ^ { \delta } } { s ^ { \delta } } \left[\left.\sum_{k=0}^{m-1} \frac{s^{\delta-k-1}}{u^{\delta-k}} \frac{\partial^{k} \mu(\xi, \zeta, \eta)}{\partial^{k} \eta}\right|_{\eta=0}\right.\right. \\
& \left.\left.-S\left[\mathscr{G}_{1}(\xi, \zeta, \eta)\right]-S\left\{\overline{\mathscr{G}}_{1}(\mu, \nu)+\mathcal{N}_{1}(\mu, \nu)\right\}\right]\right], \\
& v_{m+1}(\xi, \zeta, \eta) \\
= & \nu_{m}(\xi, \zeta, \eta)-S^{-1}\left[\frac { u ^ { \delta } } { s ^ { \delta } } \left[\left.\sum_{k=0}^{m-1} \frac{s^{\delta-k-1}}{u^{\delta-k}} \frac{\partial^{k} \nu(\xi, \zeta, \eta)}{\partial^{k} \eta}\right|_{\eta=0}\right.\right. \\
- & \left.\left.S\left[\mathscr{G}_{2}(\xi, \zeta, \eta)\right]-S\left\{\overline{\mathscr{G}}_{2}(\mu, \nu)+\mathcal{N}_{2}(\mu, \nu)\right\}\right]\right] .
\end{aligned}
$$

The initial value can be found as 


$$
\begin{aligned}
& \mu_{0}(\xi, \zeta, \eta)=S^{-1}\left[\frac{u^{\delta}}{s^{\delta}}\left\{\left.\sum_{k=0}^{m-1} \frac{s^{\delta-k-1}}{u^{\delta-k}} \frac{\partial^{k} \mu(\xi, \zeta, \eta)}{\partial^{k} \eta}\right|_{\eta=0}\right\}\right], \\
& \nu_{0}(\xi, \zeta, \eta)=S^{-1}\left[\frac{u^{\delta}}{s^{\delta}}\left\{\left.\sum_{k=0}^{m-1} \frac{s^{\delta-k-1}}{u^{\delta-k}} \frac{\partial^{k} \nu(\xi, \zeta, \eta)}{\partial^{k} \eta}\right|_{\eta=0}\right\}\right] .
\end{aligned}
$$

The converge of this technique is as follows $[59,60]$.

\section{The Procedure of SDM}

In this section, we discuss the SDM solution for system of FPDEs:

$$
\begin{array}{r}
D_{\eta}^{\delta} \mu(\xi, \zeta, \eta)+\overline{\mathscr{G}}_{1}(\mu, \nu)+\mathcal{N}_{1}(\mu, \nu)-\mathscr{G}_{1}(\xi, \zeta, \eta)=0, \\
D_{\eta}^{\delta} \nu(\xi, \zeta, \eta)+\overline{\mathscr{G}}_{2}(\mu, \nu)+\mathcal{N}_{2}(\mu, \nu)-\mathscr{G}_{2}(\xi, \zeta, \eta)=0, \\
m-1<\delta \leq m,
\end{array}
$$

with initial conditions

$$
\begin{aligned}
\mu(\xi, \zeta, 0) & =g_{1}(\xi, \zeta), \\
\nu(\xi, \zeta, 0) & =g_{2}(\xi, \zeta),
\end{aligned}
$$

where $D_{\eta}^{\delta}=\left(\partial^{\delta} / \partial \eta^{\delta}\right)$ is the Caputo fractional derivative of order $\delta ; \overline{\mathscr{G}}_{1}, \overline{\mathscr{G}}_{2}$ and $\mathcal{N}_{1}, \mathcal{N}_{2}$ are linear and nonlinear functions, respectively; and $\mathscr{G}_{1}, \mathscr{G}_{2}$ are source functions.

Apply Shehu transform to equation (20):

$$
\begin{aligned}
& S\left[D_{\eta}^{\delta} \mu(\xi, \zeta, \eta)\right]+S\left[\overline{\mathscr{G}}_{1}(\mu, \nu)+\mathcal{N}_{1}(\mu, \nu)\right. \\
& \left.-\mathscr{G}_{1}(\xi, \zeta, \eta)\right]=0, \\
& \quad S\left[D_{\eta}^{\delta} \nu(\xi, \zeta, \eta)\right]+S\left[\overline{\mathscr{G}}_{2}(\mu, \nu)+\mathcal{N}_{2}(\mu, \nu)\right. \\
& \left.-\mathscr{G}_{2}(\xi, \zeta, \eta)\right]=0 .
\end{aligned}
$$

Applying the differentiation property of Shehu transform, we have

$$
\begin{aligned}
S[\mu(\xi, \zeta, \eta)]= & \left.\frac{u^{\delta}}{s^{\delta}} \sum_{k=0}^{m-1} \frac{s^{\delta-k-1}}{u^{\delta-k}} \frac{\partial^{k} \mu(\xi, \zeta, \eta)}{\partial^{k} \eta}\right|_{\eta=0} \\
& \left.+\frac{u^{\delta}}{s^{\delta}} S\left[\mathscr{G}_{1}(\xi, \zeta, \eta)\right]-\frac{u^{\delta}}{s^{\delta}} S\left\{\overline{\mathscr{G}}_{1}(\mu, \nu)+\mathcal{N}_{1}(\mu, \nu)\right\}\right], \\
S[\nu(\xi, \zeta, \eta)]= & \left.\frac{u^{\delta}}{s^{\delta}} \sum_{k=0}^{m-1} \frac{s^{\delta-k-1}}{u^{\delta-k}} \frac{\partial^{k} \nu(\xi, \zeta, \eta)}{\partial^{k} \eta}\right|_{\eta=0} \\
& \left.+\frac{u^{\delta}}{s^{\delta}} S\left[\mathscr{G}_{2}(\xi, \zeta, \eta)\right]-\frac{u^{\delta}}{s^{\delta}} S\left\{\overline{\mathscr{G}}_{2}(\mu, \nu)+\mathcal{N}_{2}(\mu, \nu)\right\}\right] .
\end{aligned}
$$

SDM solution of infinite series $\mu(\xi, \zeta, \eta)$ and $\nu(\xi, \zeta, \eta)$ is as follows:

$$
\begin{aligned}
& \mu(\xi, \zeta, \eta)=\sum_{m=0}^{\infty} \mu_{m}(\xi, \zeta, \eta), \\
& \nu(\xi, \zeta, \eta)=\sum_{m=0}^{\infty} v_{m}(\xi, \zeta, \eta) .
\end{aligned}
$$

Adomian polynomials of nonlinear terms $\mathcal{N}_{1}$ and $\mathcal{N}_{2}$ are given as

$$
\begin{aligned}
& \mathcal{N}_{1}(\mu, \nu)=\sum_{m=0}^{\infty} \mathscr{A}_{m}, \\
& \mathcal{N}_{2}(\mu, \nu)=\sum_{m=0}^{\infty} \mathscr{B}_{m} .
\end{aligned}
$$

The nonlinear of Adomian polynomials can be defined as

$$
\begin{aligned}
& \mathscr{A}_{m}=\frac{1}{m !}\left[\frac{\partial^{m}}{\partial \lambda^{m}}\left\{\mathscr{N}_{1}\left(\sum_{k=0}^{\infty} \lambda^{k} \mu_{k}, \sum_{k=0}^{\infty} \lambda^{k} v_{k}\right)\right\}\right]_{\lambda=0}, \\
& \mathscr{B}_{m}=\frac{1}{m !}\left[\frac{\partial^{m}}{\partial \lambda^{m}}\left\{\mathscr{N}_{2}\left(\sum_{k=0}^{\infty} \lambda^{k} \mu_{k}, \sum_{k=0}^{\infty} \lambda^{k} v_{k}\right)\right\}\right]_{\lambda=0} .
\end{aligned}
$$
gives

Substituting equation (24) and equation (25) into (23)

$$
\begin{aligned}
& S\left[\sum_{m=0}^{\infty} \mu_{m}(\xi, \zeta, \eta)\right]=\left.\frac{u^{\delta}}{s^{\delta}} \sum_{k=0}^{m-1} \frac{s^{\delta-k-1}}{u^{\delta-k}} \frac{\partial^{k} \mu(\xi, \zeta, \eta)}{\partial^{k} \eta}\right|_{\eta=0}+\frac{u^{\delta}}{s^{\delta}} S\left\{\mathscr{G}_{1}(\xi, \zeta, \eta)\right\}-\frac{u^{\delta}}{s^{\delta}} S\left\{\overline{\mathscr{G}}_{1}\left(\sum_{m=0}^{\infty} \mu_{m}, \sum_{m=0}^{\infty} v_{m}\right)+\sum_{m=0}^{\infty} \mathscr{A}_{m}\right\} \\
& S\left[\sum_{m=0}^{\infty} v_{m}(\xi, \zeta, \eta)\right]=\left.\frac{u^{\delta}}{s^{\delta}} \sum_{k=0}^{m-1} \frac{s^{\delta-k-1}}{u^{\delta-k}} \frac{\partial^{k} \nu(\xi, \zeta, \eta)}{\partial^{k} \eta}\right|_{\eta=0}+\frac{u^{\delta}}{s^{\delta}} S\left\{\mathscr{G}_{2}(\xi, \zeta, \eta)\right\}-\frac{u^{\delta}}{s^{\delta}} S\left\{\overline{\mathscr{G}}_{2}\left(\sum_{m=0}^{\infty} \mu_{m}, \sum_{m=0}^{\infty} v_{m}\right)+\sum_{m=0}^{\infty} \mathscr{B}_{m}\right\} .
\end{aligned}
$$


Applying the inverse Shehu transformation to equation (20), we get

$$
\begin{aligned}
& \sum_{m=0}^{\infty} \mu_{m}(\xi, \zeta, \eta) \\
& \left.\left\{\mathscr{G}_{1}(\xi, \zeta, \eta)\right\}-\frac{u^{\delta}}{s^{\delta}} S\left\{\overline{\mathscr{G}}_{1}\left(\sum_{m=0}^{\infty} \mu_{m}, \sum_{m=0}^{\infty} v_{m}\right)+\sum_{m=0}^{\infty} \mathscr{A}_{m}\right\}\right], \\
& \sum_{m=0}^{\infty} \nu_{m}(\xi, \zeta, \eta)=S^{-1}\left[\left.\frac{u^{\delta}}{s^{\delta}} \sum_{k=0}^{m-1} \frac{s^{\delta-k-1}}{u^{\delta-k}} \frac{\partial^{k} \nu(\xi, \zeta, \eta)}{\partial^{k} \eta}\right|_{\eta=0}+\frac{u^{\delta}}{s^{\delta}} S\right. \\
& \left.\left\{\mathscr{G}_{2}(\xi, \zeta, \eta)\right\}-\frac{u^{\delta}}{s^{\delta}} S\left\{\overline{\mathscr{G}}_{2}\left(\sum_{m=0}^{\infty} \mu_{m}, \sum_{m=0}^{\infty} v_{m}\right)+\sum_{m=0}^{\infty} \mathscr{B}_{m}\right\}\right] .
\end{aligned}
$$

We define the following terms:

$$
\begin{aligned}
\mu_{0}(\xi, \zeta, \eta)= & S^{-1}\left[\left.\frac{u^{\delta}}{s^{\delta}} \sum_{k=0}^{m-1} \frac{s^{\delta-k-1}}{u^{\delta-k}} \frac{\partial^{k} \mu(\xi, \zeta, \eta)}{\partial^{k} \eta}\right|_{\eta=0}\right. \\
& \left.+\frac{u^{\delta}}{s^{\delta}} S\left\{\mathscr{G}_{1}(\xi, \zeta, \eta)\right\}\right], \\
v_{0}(\xi, \zeta, \eta)= & S^{-1}\left[\left.\frac{u^{\delta}}{s^{\delta}} \sum_{k=0}^{m-1} \frac{s^{\delta-k-1}}{u^{\delta-k}} \frac{\partial^{k} \nu(\xi, \zeta, \eta)}{\partial^{k} \eta}\right|_{\eta=0}\right. \\
& \left.+\frac{u^{\delta}}{s^{\delta}} S\left\{\mathscr{G}_{2}(\xi, \zeta, \eta)\right\}\right], \\
\mu_{1}(\xi, \zeta, \eta)= & -S^{-1}\left[\frac{u^{\delta}}{s^{\delta}} S\left\{\overline{\mathscr{G}}_{1}\left(\mu_{0}, \nu_{0}\right)+\mathscr{A}_{0}\right\}\right], \\
v_{1}(\xi, \zeta, \eta)= & -S^{-1}\left[\frac{u^{\delta}}{s^{\delta}} S\left\{\overline{\mathscr{G}}_{2}\left(\mu_{0}, v_{0}\right)+\mathscr{B}_{0}\right\}\right],
\end{aligned}
$$

in general for $m \geq 1$, and we have

$$
\begin{aligned}
& \mu_{m+1}(\xi, \zeta, \eta)=-S^{-1}\left[\frac{u^{\delta}}{s^{\delta}} S\left\{\overline{\mathscr{G}}_{1}\left(\mu_{m}, v_{m}\right)+\mathscr{A}_{m}\right\}\right], \\
& v_{m+1}(\xi, \zeta, \eta)=-S^{-1}\left[\frac{u^{\delta}}{s} S\left\{\overline{\mathscr{G}}_{2}\left(\mu_{m}, v_{m}\right)+\mathscr{B}_{m}\right\}\right] .
\end{aligned}
$$

\section{Implementation of the Methods}

Example 1. Consider fractional-order system of nonlinear equations of unsteady flow of a polytropic gas [36, 38]:

$$
\begin{array}{r}
D_{\eta}^{\delta} \mu+\mu \frac{\partial \mu}{\partial \xi}+\nu \frac{\partial \mu}{\partial \zeta}+\frac{1}{\omega} \frac{\partial \psi}{\partial \xi}=0, \\
D_{\eta}^{\delta} \nu+\mu \frac{\partial \nu}{\partial \xi}+\nu \frac{\partial \nu}{\partial \zeta}+\frac{1}{\omega} \frac{\partial \psi}{\partial \zeta}=0, \\
D_{\eta}^{\delta} \omega+\mu \frac{\partial \omega}{\partial \xi}+\nu \frac{\partial \omega}{\partial \zeta}+\omega\left(\frac{\partial \mu}{\partial \xi}+\frac{\partial \nu}{\partial \zeta}\right)=0, \\
D_{\eta}^{\delta} \psi+\mu \frac{\partial \psi}{\partial \xi}+\nu \frac{\partial \psi}{\partial \zeta}+\tau \psi\left(\frac{\partial \mu}{\partial \xi}+\frac{\partial \nu}{\partial \zeta}\right)=0,
\end{array}
$$

with initial conditions

$$
\begin{aligned}
& \mu(\xi, \zeta, 0)=e^{\xi+\zeta}, \\
& \nu(\xi, \zeta, 0)=-1-e^{\xi+\zeta}, \\
& \omega(\xi, \zeta, 0)=e^{\xi+\zeta}, \\
& \psi(\xi, \zeta, 0)=c,
\end{aligned}
$$

where $c$ is the real constant.

First, SDM is used to solve equation (31).

For this applying Shehu transformation to equation (31),

$$
\begin{aligned}
& S\left\{\frac{\partial^{\delta} \mu}{\partial \eta^{\delta}}\right\}=S\left[-\left\{\mu \frac{\partial \mu}{\partial \xi}+\nu \frac{\partial \mu}{\partial \zeta}+\frac{1}{\omega} \frac{\partial \psi}{\partial \xi}\right\}\right], \\
& S\left\{\frac{\partial^{\delta} \nu}{\partial \eta^{\delta}}\right\}=S\left[-\left\{\mu \frac{\partial \nu}{\partial \xi}+\nu \frac{\partial \nu}{\partial \zeta}+\frac{1}{\omega} \frac{\partial \psi}{\partial \zeta}\right\}\right] \text {, } \\
& S\left\{\frac{\partial^{\delta} \omega}{\partial \eta^{\delta}}\right\}=S\left[-\left\{\mu \frac{\partial \omega}{\partial \xi}+\nu \frac{\partial \omega}{\partial \zeta}+\omega\left(\frac{\partial \mu}{\partial \xi}+\frac{\partial \nu}{\partial \zeta}\right),\right\}\right] \\
& S\left\{\frac{\partial^{\delta} \psi}{\partial \eta^{\delta}}\right\}=S\left[-\left\{\mu \frac{\partial \psi}{\partial \xi}+\nu \frac{\partial \psi}{\partial \zeta}+\tau \psi\left(\frac{\partial \mu}{\partial \xi}+\frac{\partial \nu}{\partial \zeta}\right),\right\}\right] \\
& \frac{s^{\delta}}{u^{\delta}} S\{\mu(\xi, \zeta, \eta)\}-\frac{s^{\delta-1}}{u^{\delta}} \mu(\xi, \zeta, 0) \\
& =S\left[-\left\{\mu \frac{\partial \mu}{\partial \xi}+\nu \frac{\partial \mu}{\partial \zeta}+\frac{1}{\omega} \frac{\partial \psi}{\partial \xi}\right\}\right], \\
& \frac{s^{\delta}}{u^{\delta}} S\{\nu(\xi, \zeta, \eta)\}-\frac{s^{\delta-1}}{u^{\delta}} \nu(\xi, \zeta, 0) \\
& =S\left[-\left\{\mu \frac{\partial \nu}{\partial \xi}+\nu \frac{\partial \nu}{\partial \zeta}+\frac{1}{\omega} \frac{\partial \psi}{\partial \zeta}\right\}\right], \\
& \frac{s^{\delta}}{u^{\delta}} S\{\omega(\xi, \zeta, \eta)\}-\frac{s^{\delta-1}}{u^{\delta}} \omega(\xi, \zeta, 0) \\
& =S\left[-\left\{\mu \frac{\partial \omega}{\partial \xi}+\nu \frac{\partial \omega}{\partial \zeta}+\omega\left(\frac{\partial \mu}{\partial \xi}+\frac{\partial \nu}{\partial \zeta}\right)\right\}\right], \\
& \frac{s^{\delta}}{u^{\delta}} S\{\psi(\xi, \zeta, \eta)\}-\frac{s^{\delta-1}}{u^{\delta}} \psi(\xi, \zeta, 0) \\
& =S\left[-\left\{\mu \frac{\partial \psi}{\partial \xi}+\nu \frac{\partial \psi}{\partial \zeta}+\tau \psi\left(\frac{\partial \mu}{\partial \xi}+\frac{\partial \nu}{\partial \zeta}\right)\right\}\right] \text {. }
\end{aligned}
$$


The above algorithm is reduced to simplified form:

$S\{\mu(\xi, \zeta, \eta)\}=\frac{1}{s}\{\mu(\xi, \zeta, 0)\}$

$$
-\frac{u^{\delta}}{s^{\delta}} S\left[\left\{\mu \frac{\partial \mu}{\partial \xi}+\nu \frac{\partial \mu}{\partial \zeta}+\frac{1}{\omega} \frac{\partial \psi}{\partial \xi}\right\}\right],
$$

$S\{\nu(\xi, \zeta, \eta)\}=\frac{1}{s}\{\nu(\xi, \zeta, 0)\}$

$$
-\frac{u^{\delta}}{s} S\left[\left\{\mu \frac{\partial \nu}{\partial \xi}+\nu \frac{\partial \nu}{\partial \zeta}+\frac{1}{\omega} \frac{\partial \psi}{\partial \zeta}\right\}\right],
$$

$S\{\omega(\xi, \zeta, \eta)\}=\frac{1}{s}\{\omega(\xi, \zeta, 0)\}$

$$
-\frac{u^{\delta}}{s^{\delta}} S\left[\left\{\mu \frac{\partial \omega}{\partial \xi}+\nu \frac{\partial \omega}{\partial \zeta}+\omega\left(\frac{\partial \mu}{\partial \xi}+\frac{\partial \nu}{\partial \zeta}\right)\right\}\right],
$$

$S\{\psi(\xi, \zeta, \eta)\}=\frac{1}{s}\{\psi(\xi, \zeta, 0)\}$

$$
-\frac{u^{\delta}}{s} S\left[\left\{\mu \frac{\partial \psi}{\partial \xi}+\nu \frac{\partial \psi}{\partial \zeta}+\tau \psi\left(\frac{\partial \mu}{\partial \xi}+\frac{\partial \nu}{\partial \zeta}\right)\right\}\right] .
$$

Applying inverse Shehu transformation, we get

$$
\begin{aligned}
\mu(\xi, \zeta, \eta)= & \mu(\xi, \zeta, 0)- \\
& S^{-1}\left[\frac{u^{\delta}}{s^{\delta}} S\left\{\mu \frac{\partial \mu}{\partial \xi}+\nu \frac{\partial \mu}{\partial \zeta}+\frac{1}{\omega} \frac{\partial \psi}{\partial \xi}\right\}\right] \\
\nu(\xi, \zeta, \eta)= & \nu(\xi, \zeta, 0)- \\
& S^{-1}\left[\frac{u^{\delta}}{s^{\delta}} S\left\{\mu \frac{\partial \nu}{\partial \xi}+\nu \frac{\partial \nu}{\partial \zeta}+\frac{1}{\omega} \frac{\partial \psi}{\partial \zeta}\right\}\right] \\
\omega(\xi, \zeta, \eta)= & \omega(\xi, \zeta, 0)- \\
& S^{-1}\left[\frac{u^{\delta}}{s^{\delta}} S\left\{\mu \frac{\partial \omega}{\partial \xi}+\nu \frac{\partial \omega}{\partial \zeta}+\omega\left(\frac{\partial \mu}{\partial \xi}+\frac{\partial \nu}{\partial \zeta}\right)\right\}\right] \\
\psi(\xi, \zeta, \eta)= & \psi(\xi, \zeta, 0)- \\
& S^{-1}\left[\frac{u^{\delta}}{s^{\delta}} S\left\{\mu \frac{\partial \psi}{\partial \xi}+\nu \frac{\partial \psi}{\partial \zeta}+\tau \psi\left(\frac{\partial \mu}{\partial \xi}+\frac{\partial \nu}{\partial \zeta}\right)\right\}\right]
\end{aligned}
$$

Equation (35) can be written in an operator form as

$$
\begin{aligned}
\mu(\xi, \zeta, \eta)= & \mu(\xi, \zeta, 0)-S^{-1}\left[\frac{u^{\delta}}{s^{\delta}} S\right. \\
& \left.\left\{A_{1}\left(\mu, \mu_{\xi}\right)+B_{1}\left(\nu, \mu_{\zeta}\right)+C_{1}\left(\omega, \psi_{\xi}\right)\right\}\right],
\end{aligned}
$$

$$
v(\xi, \zeta, \eta)=v(\xi, \zeta, 0)-S^{-1}\left[\frac{u^{\delta}}{s^{\delta}} S\right.
$$

$$
\left.\left\{A_{2}\left(\mu, \nu_{\xi}\right)+B_{2}\left(\nu, \nu_{\zeta}\right)+C_{2}\left(\omega, \psi_{\zeta}\right)\right\}\right]
$$

$$
\begin{aligned}
\omega(\xi, \zeta, \eta)= & \omega(\xi, \zeta, 0)-S^{-1}\left[\frac { u ^ { \delta } } { s } S \left\{A_{3}\left(\mu, \omega_{\xi}\right)+\right.\right. \\
& \left.\left.B_{3}\left(\nu, \omega_{\zeta}\right)+C_{3}\left(\omega, \mu_{\xi}\right)+D_{3}\left(\omega, \nu_{\zeta}\right)\right\}\right]
\end{aligned}
$$

$\psi(\xi, \zeta, \eta)=\psi(\xi, \zeta, 0)-S^{-1}\left[\frac{u^{\delta}}{s^{\delta}} S\left\{A_{4}\left(\mu, \psi_{\xi}\right)+\right]\right.$

$$
\left.\left.B_{4}\left(\nu, \psi_{\zeta}\right)+\tau C_{4}\left(\psi, \mu_{\xi}\right)+\tau D_{4}\left(\psi, \nu_{\zeta}\right)\right\}\right] .
$$

Assume that the unknown functions $\mu(\xi, \zeta, \eta), v(\xi, \zeta, \eta)$, $\omega(\xi, \zeta, \eta)$, and $\psi(\xi, \zeta, \eta)$ have infinite series solution as follows:

$$
\begin{aligned}
& \mu(\xi, \zeta, \eta)=\sum_{m=0}^{\infty} \mu_{m}(\xi, \zeta, \eta), \\
& \nu(\xi, \zeta, \eta)=\sum_{m=0}^{\infty} \nu_{m}(\xi, \zeta, \eta), \\
& \omega(\xi, \zeta, \eta)=\sum_{m=0}^{\infty} \omega_{m}(\xi, \zeta, \eta), \\
& \psi(\xi, \zeta, \eta)=\sum_{m=0}^{\infty} \psi_{m}(\xi, \zeta, \eta) .
\end{aligned}
$$

All forms of nonlinear Adomian polynomials can be defined as 


$$
\begin{aligned}
& A_{1}\left(\mu, \mu_{\xi}\right)=\mu_{0} \mu_{0 \xi}+\left(\mu_{1} \mu_{0 \xi}+\mu_{0} \mu_{1 \xi}\right)+\cdots, \\
& B_{1}\left(\nu, \mu_{\zeta}\right)=\nu_{0} \mu_{0 \xi}+\left(\nu_{1} \mu_{0 \xi}+v_{0} \mu_{1 \xi}\right)+\cdots, \\
& C_{1}\left(\omega, \psi_{\xi}\right)=\frac{\psi_{0 \xi}}{\omega_{0}}+\frac{\omega_{0} \psi_{1 \xi}-\omega_{1} \psi_{0 \xi}}{\omega_{0}}+\cdots, \\
& A_{2}\left(\mu, v_{\xi}\right)=\mu_{0} v_{0 \xi}+\left(\mu_{1} v_{0 \xi}+\mu_{0} v_{1 \xi}\right)+\cdots, \\
& B_{2}\left(\nu, \nu_{\zeta}\right)=\nu_{0} \nu_{0 \xi}+\left(\nu_{1} \nu_{0 \xi}+\nu_{0} \nu_{1 \xi}\right)+\cdots, \\
& C_{2}\left(\omega, \psi_{\zeta}\right)=\frac{\psi_{0 \zeta}}{\omega_{0}}+\frac{\omega_{0} \psi_{1 \zeta}-\omega_{1} \psi_{0 \zeta}}{\omega_{0}}+\cdots, \\
& A_{3}\left(\mu, \omega_{\xi}\right)=\mu_{0} \omega_{0 \xi}+\left(\mu_{1} \omega_{0 \xi}+\mu_{0} \omega_{1 \xi}\right)+\cdots, \\
& B_{3}\left(\nu, \omega_{\zeta}\right)=v_{0} \omega_{0 \xi}+\left(\nu_{1} \omega_{0 \xi}+v_{0} \omega_{1 \xi}\right)+\cdots, \\
& C_{3}\left(\omega, \mu_{\xi}\right)=\omega_{0} \mu_{0 \xi}+\left(\omega_{1} \mu_{0 \xi}+\omega_{0} \mu_{1 \xi}\right)+\cdots, \\
& D_{3}\left(\omega, \nu_{\zeta}\right)=\omega_{0} \nu_{0 \zeta}+\left(\omega_{1} \nu_{0 \zeta}+\omega_{0} \nu_{1 \zeta}\right)+\cdots, \\
& A_{4}\left(\mu, \psi_{\xi}\right)=\mu_{0} \psi_{0 \xi}+\left(\mu_{1} \psi_{0 \xi}+\mu_{0} \psi_{1 \xi}\right)+\cdots, \\
& C_{4}\left(\psi, \mu_{\xi}\right)=\psi_{0} \mu_{0 \xi}+\left(\psi_{1} \mu_{0 \xi}+\psi_{0} \mu_{1 \xi}\right)+\cdots, \\
& D_{4}\left(\psi, \nu_{\zeta}\right)=\psi_{0} \nu_{0 \zeta}+\left(\psi_{1} \nu_{0 \zeta}+\psi_{0} \nu_{1 \zeta}\right)+\cdots \text {. }
\end{aligned}
$$

The initial sources are

$$
\begin{aligned}
\mu_{0}(\xi, \zeta, \eta)= & e^{\xi+\zeta}, \\
\nu_{0}(\xi, \zeta, \eta)= & -1-e^{\xi+\zeta} \\
\omega_{0}(\xi, \zeta, \eta)= & e^{\xi+\zeta}, \\
\psi_{0}(\xi, \zeta, 0)= & c . \\
\mu_{m}(\xi, \zeta, \eta)= & -S^{-1}\left[\frac{u^{\delta}}{s^{\delta}} S\right. \\
& \left.\cdot\left\{A_{1}\left(\mu, \mu_{\xi}\right)+B_{1}\left(\nu, \mu_{\zeta}\right)+C_{1}\left(\omega, \psi_{\xi}\right)\right\}\right] \\
\nu_{m}(\xi, \zeta, \eta)= & -S^{-1}\left[\frac{u^{\delta}}{s^{\delta}} S\right. \\
& \left.\cdot\left\{A_{2}\left(\mu, \nu_{\xi}\right)+B_{2}\left(\nu, \nu_{\zeta}\right)+C_{2}\left(\omega, \psi_{\zeta}\right)\right\}\right], \\
& \left.\left.B_{4}\left(\nu, \psi_{\zeta}\right)+\tau C_{4}\left(\psi, \mu_{\xi}\right)+\tau D_{4}\left(\psi, \nu_{\zeta}\right)\right\}\right] \\
& \left.\left.B_{3}\left(\nu, \omega_{\zeta}\right)+C_{3}\left(\omega, \mu_{\xi}\right)+D_{3}\left(\omega, \nu_{\zeta}\right)\right\}\right], \\
\omega_{m}(\xi, \zeta, \eta)= & -S^{-1}\left[\frac { u ^ { \delta } } { s ^ { \delta } } S \left\{A_{3}\left(\mu, \omega_{\xi}\right)+\right.\right. \\
& -1\left[\frac { u ^ { \delta } } { s ^ { \delta } } S \left\{A_{4}\left(\mu, \psi_{\xi}\right)+\right.\right.
\end{aligned}
$$

For $m=1$,

$$
\begin{aligned}
& \mu_{1}(\xi, \zeta, \eta)=e^{\xi+\zeta} \frac{\eta^{\delta}}{\Gamma(\delta+1)}, \\
& \nu_{1}(\xi, \zeta, \eta)=-e^{\xi+\zeta} \frac{\eta^{\delta}}{\Gamma(\delta+1)}, \\
& \omega_{1}(\xi, \zeta, \eta)=e^{\xi+\zeta} \frac{\eta^{\delta}}{\Gamma(\delta+1)}, \\
& \psi_{1}(\xi, \zeta, 0)=0 .
\end{aligned}
$$

For $m=2$,

$$
\begin{aligned}
& \mu_{2}(\xi, \zeta, \eta)=e^{\xi+\zeta} \frac{\eta^{2 \delta}}{\Gamma(2 \delta+1)} \\
& \nu_{2}(\xi, \zeta, \eta)=-e^{\xi+\zeta} \frac{\eta^{2 \delta}}{\Gamma(2 \delta+1)} \\
& \omega_{2}(\xi, \zeta, \eta)=e^{\xi+\zeta} \frac{\eta^{2 \delta}}{\Gamma(2 \delta+1)} \\
& \psi_{2}(\xi, \zeta, 0)=0
\end{aligned}
$$

For $m=3$,

$$
\begin{aligned}
& \mu_{3}(\xi, \zeta, \eta)=e^{\xi+\zeta} \frac{\eta^{3 \delta}}{\Gamma(3 \delta+1)}, \\
& v_{3}(\xi, \zeta, \eta)=-e^{\xi+\zeta} \frac{\eta^{3 \delta}}{\Gamma(3 \delta+1)}, \\
& \omega_{3}(\xi, \zeta, \eta)=e^{\xi+\zeta} \frac{\eta^{3 \delta}}{\Gamma(3 \delta+1)}, \\
& \psi_{3}(\xi, \zeta, 0)=0 \text {. } \\
& \mu_{m}(\xi, \zeta, \eta)=\mu_{0}(\xi, \zeta, \eta)+\mu_{1}(\xi, \zeta, \eta)+\cdots, \\
& \nu_{m}(\xi, \zeta, \eta)=\nu_{0}(\xi, \zeta, \eta)+\nu_{1}(\xi, \zeta, \eta)+\cdots, \\
& v_{m}(\xi, \zeta, \eta)=v_{0}(\xi, \zeta, \eta)+v_{1}(\xi, \zeta, \eta)+\cdots, \\
& \omega_{m}(\xi, \zeta, \eta)=\omega_{0}(\xi, \zeta, \eta)+\omega_{1}(\xi, \zeta, \eta)+\cdots, \\
& \psi_{m}(\xi, \zeta, 0)=\psi_{0}(\xi, \zeta, \eta)+\psi_{1}(\xi, \zeta, \eta)+\cdots, \\
& \mu(\xi, \zeta, \eta)=e^{\xi+\zeta}+e^{\xi+\zeta} \frac{\eta^{\delta}}{\Gamma(\delta+1)} \\
& +e^{\xi+\zeta} \frac{\eta^{2 \delta}}{\Gamma(2 \delta+1)}+e^{\xi+\zeta} \frac{\eta^{3 \delta}}{\Gamma(3 \delta+1)}+\cdots, \\
& \nu(\xi, \zeta, \eta)=-1-e^{\xi+\zeta}-e^{\xi+\zeta} \frac{\eta^{\delta}}{\Gamma(\delta+1)} \\
& -e^{\xi+\zeta} \frac{\eta^{2 \delta}}{\Gamma(2 \delta+1)}-e^{\xi+\zeta} \frac{\eta^{3 \delta}}{\Gamma(3 \delta+1)}-\cdots, \\
& \omega(\xi, \zeta, \eta)=e^{\xi+\zeta}+e^{\xi+\zeta} \frac{\eta^{\delta}}{\Gamma(\delta+1)} \\
& +e^{\xi+\zeta} \frac{\eta^{2 \delta}}{\Gamma(2 \delta+1)}+e^{\xi+\zeta} \frac{\eta^{3 \delta}}{\Gamma(3 \delta+1)}+\cdots,
\end{aligned}
$$$$
\psi(\xi, \zeta, 0)=c+0+\cdots \text {. }
$$

In general, we have 


$$
\begin{aligned}
& \mu_{m}(\xi, \zeta, \eta)=e^{\xi+\zeta} \frac{\eta^{m \delta}}{\Gamma(m \delta+1)} \\
& v_{m}(\xi, \zeta, \eta)=-e^{\xi+\zeta} \frac{\eta^{m \delta}}{\Gamma(m \delta+1)} \\
& \omega_{m}(\xi, \zeta, \eta)=e^{\xi+\zeta} \frac{\eta^{m \delta}}{\Gamma(m \delta+1)}, \\
& \psi_{m}(\xi, \zeta, 0)=0, \quad m=1,2, \ldots
\end{aligned}
$$

The Approximate Solution by VITM

According to equation (16) and the iteration formulas for system (31), we get

$$
\begin{aligned}
& \mu_{m+1}(\xi, \zeta, \eta)=\mu_{m}(\xi, \zeta, \eta)-N^{-}\left[\frac { u ^ { \delta } } { s ^ { \delta } } S \left\{\frac{s^{\delta}}{u^{\delta}}\right.\right. \\
& \left.\left.\cdot \frac{\partial \mu_{m}}{\partial \eta}+\mu_{m} \frac{\partial \mu_{m}}{\partial \xi}+v_{m} \frac{\partial \mu_{m}}{\partial \zeta}+\frac{1}{\omega_{m}} \frac{\partial \psi_{m}}{\partial \xi}\right\}\right] \\
& v_{m+1}(\xi, \zeta, \eta)=v_{m}(\xi, \zeta, \eta)-S^{-1}\left[\frac { u ^ { \delta } } { s ^ { \delta } } S \left\{\frac{s^{\delta}}{u^{\delta}}\right.\right. \\
& \left.\left.\cdot \frac{\partial v_{m}}{\partial \eta}+\mu_{m} \frac{\partial v_{m}}{\partial \xi}+v_{m} \frac{\partial v_{m}}{\partial \zeta}+\frac{1}{\omega_{m}} \frac{\partial \psi_{m}}{\partial \zeta}\right\}\right] \\
& \omega_{m+1}(\xi, \zeta, \eta)=\omega_{m}(\xi, \zeta, \eta)-N^{-}\left[\frac{u^{\delta}}{s^{\delta}} S\left\{\frac{s^{\delta}}{u^{\delta}} \frac{\partial \mu_{m}}{\partial \eta}\right]\right. \\
& \left.\left.+\mu_{m} \frac{\partial \omega_{m}}{\partial \xi}+v_{m} \frac{\partial \omega_{m}}{\partial \zeta}+\omega_{m}\left(\frac{\partial \mu_{m}}{\partial \xi}+\frac{\partial \nu_{m}}{\partial \zeta}\right)\right\}\right] \\
& \psi_{m+1}(\xi, \zeta, \eta)=\psi_{m}(\xi, \zeta, \eta)-S^{-1}\left[\frac { u ^ { \delta } } { s ^ { \delta } } S \left\{\frac{s^{\delta}}{u^{\delta}} \frac{\partial v_{m}}{\partial \eta}\right.\right. \\
& \left.\left.+\mu_{m} \frac{\partial \psi_{m}}{\partial \xi}+\nu_{m} \frac{\partial \psi_{m}}{\partial \zeta}+\tau \psi_{m}\left(\frac{\partial \mu_{m}}{\partial \xi}+\frac{\partial \nu_{m}}{\partial \zeta}\right)\right\}\right],
\end{aligned}
$$

where

$$
\begin{aligned}
& \mu_{0}(\xi, \zeta, \eta)=e^{\xi+\zeta}, \\
& v_{0}(\xi, \zeta, \eta)=-1-e^{\xi+\zeta}, \\
& \omega_{0}(\xi, \zeta, \eta)=e^{\xi+\zeta} \\
& \psi_{0}(\xi, \zeta, 0)=c .
\end{aligned}
$$

For $m=0,1,2, \ldots$,

$$
\begin{aligned}
\mu_{1}(\xi, \zeta, \eta)= & \mu_{0}(\xi, \zeta, \eta)- \\
& N^{-}\left[\frac{u^{\delta}}{s^{\delta}} S\left\{\frac{s^{\delta}}{u^{\delta}} \frac{\partial \mu_{0}}{\partial \eta}+\mu_{0} \frac{\partial \mu_{0}}{\partial \xi}+\nu_{0} \frac{\partial \mu_{0}}{\partial \zeta}+\frac{1}{\omega_{0}} \frac{\partial \psi_{0}}{\partial \xi}\right\}\right],
\end{aligned}
$$$$
\nu_{1}(\xi, \zeta, \eta)=v_{0}(\xi, \zeta, \eta)-
$$$$
S^{-1}\left[\frac{u^{\delta}}{s^{\delta}} S\left\{\frac{s^{\delta}}{u^{\delta}} \frac{\partial v_{0}}{\partial \eta}+\mu_{0} \frac{\partial v_{0}}{\partial \xi}+v_{0} \frac{\partial v_{0}}{\partial \zeta}+\frac{1}{\omega_{0}} \frac{\partial \psi_{0}}{\partial \zeta}\right\}\right],
$$$$
\omega_{1}(\xi, \zeta, \eta)=\omega_{0}(\xi, \zeta, \eta)-N^{-}\left[\frac { u ^ { \delta } } { s ^ { \delta } } S \left\{\frac{s^{\delta}}{u^{\delta}}\right.\right.
$$$$
\left.\left.\cdot \frac{\partial \mu_{0}}{\partial \eta}+\mu_{0} \frac{\partial \omega_{0}}{\partial \xi_{0}}+v_{0} \frac{\partial \omega_{0}}{\partial \zeta_{0}}+\omega_{0}\left(\frac{\partial \mu_{0}}{\partial \xi}+\frac{\partial \nu_{0}}{\partial \zeta}\right)\right\}\right] \text {, }
$$

$\psi_{1}(\xi, \zeta, \eta)=\psi_{0}(\xi, \zeta, \eta)-S^{-1}\left[\frac{u^{\delta}}{s^{\delta}} S\left\{\frac{s^{\delta}}{u^{\delta}}\right.\right.$

$$
\left.\left.\cdot \frac{\partial v_{0}}{\partial \eta}+\mu_{0} \frac{\partial \psi_{0}}{\partial \xi}+v_{0} \frac{\partial \psi_{0}}{\partial \zeta}+\tau \psi_{0}\left(\frac{\partial \mu_{0}}{\partial \xi}+\frac{\partial \nu_{0}}{\partial \zeta}\right)\right\}\right]
$$

$\mu_{1}(\xi, \zeta, \eta)=e^{\xi+\zeta}\left\{1+\frac{\eta^{\delta}}{\Gamma(\delta+1)}\right\}$,

$$
\nu_{1}(\xi, \zeta, \eta)=-1-e^{\xi+\zeta}\left\{1+\frac{\eta^{\delta}}{\Gamma(\delta+1)}\right\} \text {, }
$$$$
\omega_{1}(\xi, \zeta, \eta)=e^{\xi+\zeta}\left\{1+\frac{\eta^{\delta}}{\Gamma(\delta+1)}\right\},
$$

$\psi_{1}(\xi, \zeta, 0)=c+0$.

$\mu_{2}(\xi, \zeta, \eta)=\mu_{1}(\xi, \zeta, \eta)-$

$$
N^{-}\left[\frac{u^{\delta}}{s^{\delta}} S\left\{\frac{s^{\delta}}{u^{\delta}} \frac{\partial \mu_{1}}{\partial \eta}+\mu_{1} \frac{\partial \mu_{1}}{\partial \xi}+\nu_{1} \frac{\partial \mu_{1}}{\partial \zeta}+\frac{1}{\omega_{1}} \frac{\partial \psi_{1}}{\partial \xi}\right\}\right],
$$

$v_{2}(\xi, \zeta, \eta)=v_{1}(\xi, \zeta, \eta)-$

$$
S^{-1}\left[\frac{u^{\delta}}{s^{\delta}} S\left\{\frac{s^{\delta}}{u^{\delta}} \frac{\partial \nu_{1}}{\partial \eta}+\mu_{1} \frac{\partial \nu_{1}}{\partial \xi}+v_{1} \frac{\partial \nu_{1}}{\partial \zeta}+\frac{1}{\omega_{1}} \frac{\partial \psi_{1}}{\partial \zeta}\right\}\right],
$$

$\omega_{2}(\xi, \zeta, \eta)=\omega_{1}(\xi, \zeta, \eta)-N^{-}\left[\frac{u^{\delta}}{s^{\delta}} S\left\{\frac{s^{\delta}}{u^{\delta}}\right.\right.$

$$
\begin{gathered}
\left.\left.\cdot \frac{\partial \mu_{1}}{\partial \eta}+\mu_{1} \frac{\partial \omega_{1}}{\partial \xi_{0}}+\nu_{1} \frac{\partial \omega_{1}}{\partial \zeta_{1}}+\omega_{1}\left(\frac{\partial \mu_{1}}{\partial \xi}+\frac{\partial \nu_{1}}{\partial \zeta}\right)\right\}\right] \\
\psi_{2}(\xi, \zeta, \eta)=\psi_{1}(\xi, \zeta, \eta)-S^{-1}\left[\frac { u ^ { \delta } } { s ^ { \delta } } S \left\{\frac{s^{\delta}}{u^{\delta}}\right.\right. \\
\left.\left.\cdot \frac{\partial \nu_{1}}{\partial \eta}+\mu_{1} \frac{\partial \psi_{1}}{\partial \xi}+\nu_{1} \frac{\partial \psi_{1}}{\partial \zeta^{\prime}}+\tau \psi_{1}\left(\frac{\partial \mu_{1}}{\partial \xi}+\frac{\partial \nu_{1}}{\partial \zeta}\right)\right\}\right] \\
\mu_{2}(\xi, \zeta, \eta)=e^{\xi+\zeta}\left\{1+\frac{\eta^{2 \delta}}{\Gamma(\delta+1)}+\frac{\eta^{\prime}}{\Gamma(2 \delta+1)}\right\}
\end{gathered}
$$




$$
\begin{aligned}
& \nu_{2}(\xi, \zeta, \eta)=-1-e^{\xi+\zeta}\left\{1+\frac{\eta^{\delta}}{\Gamma(\delta+1)}+\frac{\eta^{2 \delta}}{\Gamma(2 \delta+1)}\right\}, \\
& \omega_{2}(\xi, \zeta, \eta)=e^{\xi+\zeta}\left\{1+\frac{\eta^{\delta}}{\Gamma(\delta+1)}+\frac{\eta^{2 \delta}}{\Gamma(2 \delta+1)}\right\}, \\
& \psi_{2}(\xi, \zeta, 0)=c+0 \text {. } \\
& \mu_{3}(\xi, \zeta, \eta)=\mu_{2}(\xi, \zeta, \eta)- \\
& N^{-}\left[\frac{u^{\delta}}{s^{\delta}} S\left\{\frac{s^{\delta}}{u^{\delta}} \frac{\partial \mu_{2}}{\partial \eta}+\mu_{2} \frac{\partial \mu_{2}}{\partial \xi}+v_{2} \frac{\partial \mu_{2}}{\partial \zeta}+\frac{1}{\omega_{2}} \frac{\partial \psi_{2}}{\partial \xi}\right\}\right], \\
& v_{3}(\xi, \zeta, \eta)=v_{2}(\xi, \zeta, \eta)- \\
& S^{-1}\left[\frac{u^{\delta}}{s^{\delta}} S\left\{\frac{s^{\delta}}{u^{\delta}} \frac{\partial \nu_{2}}{\partial \eta}+\mu_{2} \frac{\partial \nu_{2}}{\partial \xi}+v_{2} \frac{\partial \nu_{2}}{\partial \zeta}+\frac{1}{\omega_{2}} \frac{\partial \psi_{2}}{\partial \zeta}\right\}\right], \\
& \omega_{3}(\xi, \zeta, \eta)=\omega_{2}(\xi, \zeta, \eta)-N^{-}\left[\frac { u ^ { \delta } } { s ^ { \delta } } S \left\{\frac{s^{\delta}}{u^{\delta}}\right.\right. \\
& \left.\left.\frac{\partial \mu_{2}}{\partial \eta}+\mu_{2} \frac{\partial \omega_{2}}{\partial \xi_{0}}+v_{2} \frac{\partial \omega_{2}}{\partial \zeta_{2}}+\omega_{2}\left(\frac{\partial \mu_{2}}{\partial \xi}+\frac{\partial \nu_{2}}{\partial \zeta}\right)\right\}\right], \\
& \psi_{3}(\xi, \zeta, \eta)=\psi_{2}(\xi, \zeta, \eta)-S^{-1}\left[\frac { u ^ { \delta } } { s ^ { \delta } } S \left\{\frac{s^{\delta}}{u^{\delta}}\right.\right. \\
& \left.\left.\cdot \frac{\partial \nu_{2}}{\partial \eta}+\mu_{2} \frac{\partial \psi_{2}}{\partial \xi}+v_{2} \frac{\partial \psi_{2}}{\partial \zeta}+\tau \psi_{2}\left(\frac{\partial \mu_{2}}{\partial \xi}+\frac{\partial \nu_{2}}{\partial \zeta}\right)\right\}\right] \text {, } \\
& \mu_{3}(\xi, \zeta, \eta)=e^{\xi+\zeta}\left\{1+\frac{\eta^{\delta}}{\Gamma(\delta+1)}\right. \\
& \left.+\frac{\eta^{2 \delta}}{\Gamma(2 \delta+1)}+\frac{\eta^{3 \delta}}{\Gamma(3 \delta+1)}\right\} \text {, } \\
& \omega_{3}(\xi, \zeta, \eta) \\
& \left.+\frac{\eta^{2 \delta}}{\Gamma(2 \delta+1)}+\frac{\eta^{3 \delta}}{\Gamma(3 \delta+1)}\right\} \text {, } \\
& \psi_{3}(\xi, \zeta, 0)=c+0 \text {. } \\
& \nu_{3}(\xi, \zeta, \eta)=-1-e^{\xi+\zeta}\left\{1+\frac{\eta^{\delta}}{\Gamma(\delta+1)}\right. \\
& \left.+\frac{\eta^{2 \delta}}{\Gamma(2 \delta+1)}+\frac{\eta^{3 \delta}}{\Gamma(3 \delta+1)}\right\} \\
& v(\xi, \zeta, \eta)=\sum_{m=0}^{\infty} v_{m}(\xi, \zeta) \\
& \left.+\frac{\eta^{2 \delta}}{\Gamma(2 \delta+1)}+\frac{\eta^{3 \delta}}{\Gamma(3 \delta+1)}+\cdots+\frac{\eta^{m \delta}}{\Gamma(m \delta+1)}\right\}, \\
& \omega(\xi, \zeta, \eta)=\sum_{m=0}^{\infty} \omega_{m}(\xi, \zeta) \\
& \left.+\frac{\eta^{2 \delta}}{\Gamma(2 \delta+1)}+\frac{\eta^{3 \delta}}{\Gamma(3 \delta+1)}+\cdots+\frac{\eta^{m \delta}}{\Gamma(m \delta+1)}\right\}, \\
& \psi(\xi, \zeta, 0)=\sum_{m=0}^{\infty} \psi_{m}(\xi, \zeta) \\
& \mu(\xi, \zeta, \eta)=\sum_{m=0}^{\infty} \mu_{m}(\xi, \zeta) \\
& \left.+\frac{\eta^{2 \delta}}{\Gamma(2 \delta+1)}+\frac{\eta^{3 \delta}}{\Gamma(3 \delta+1)}+\cdots+\frac{\eta^{m \delta}}{\Gamma(m \delta+1)}\right\} .
\end{aligned}
$$

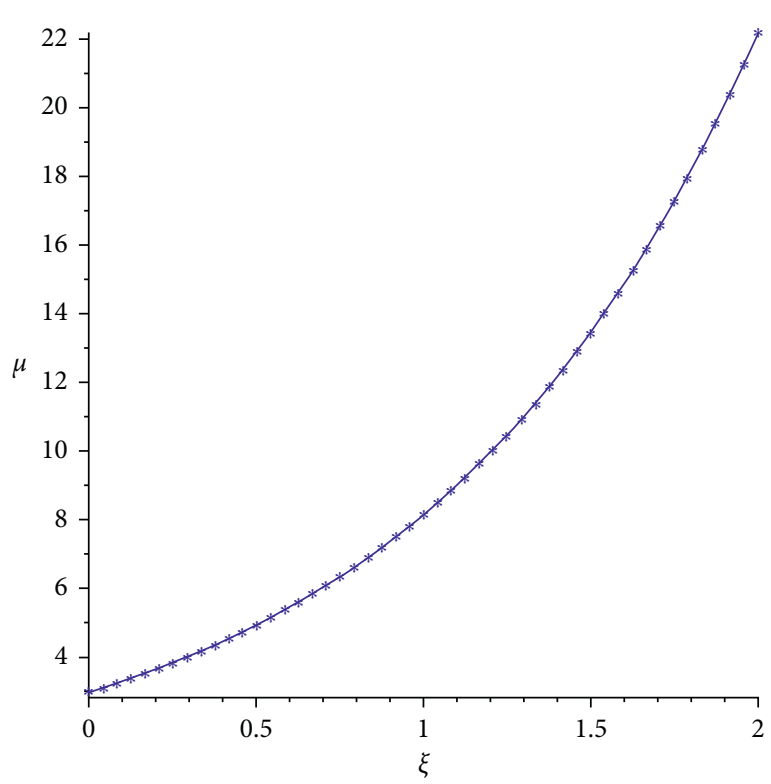

FIGURE 1: SDM and VITM solution graph of $\mu$ at $\zeta=1$ and $\eta=0.5$ of Example 1.

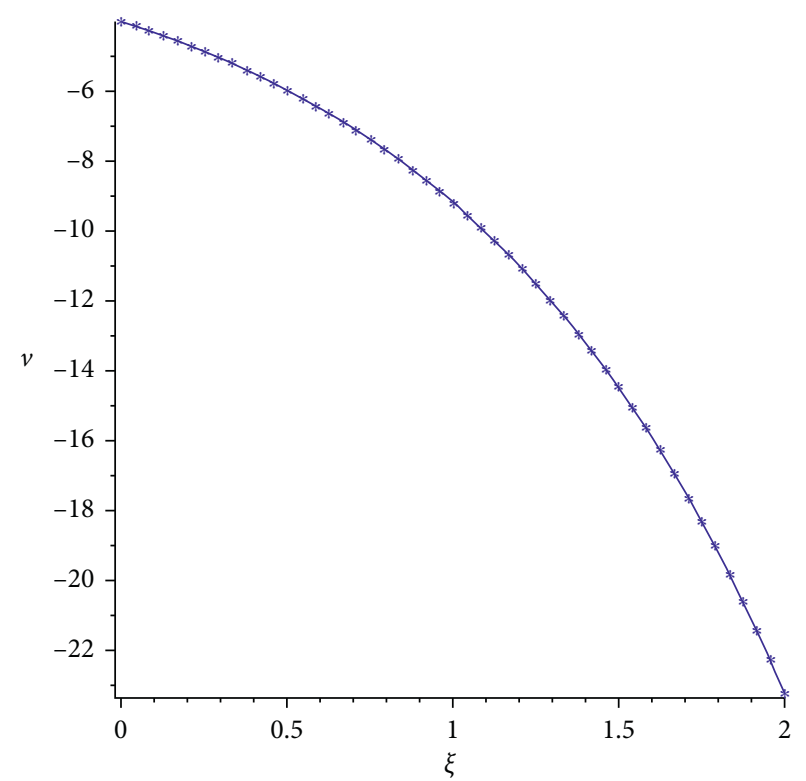

FIGURE 2: SDM and VITM solution graph of $\nu$ at $\zeta=1$ and $\eta=0.5$ of Example 1.

The exact solution of equation (31) at $\delta=1$ is

$$
\begin{aligned}
& \mu(\xi, \zeta, \eta)=e^{\xi+\zeta+\eta}, \\
& \nu(\xi, \zeta, \eta)=-1-e^{\xi+\zeta+\eta}, \\
& \omega(\xi, \zeta, \eta)=e^{\xi+\zeta+\eta}, \\
& \psi(\xi, \zeta, 0)=c .
\end{aligned}
$$




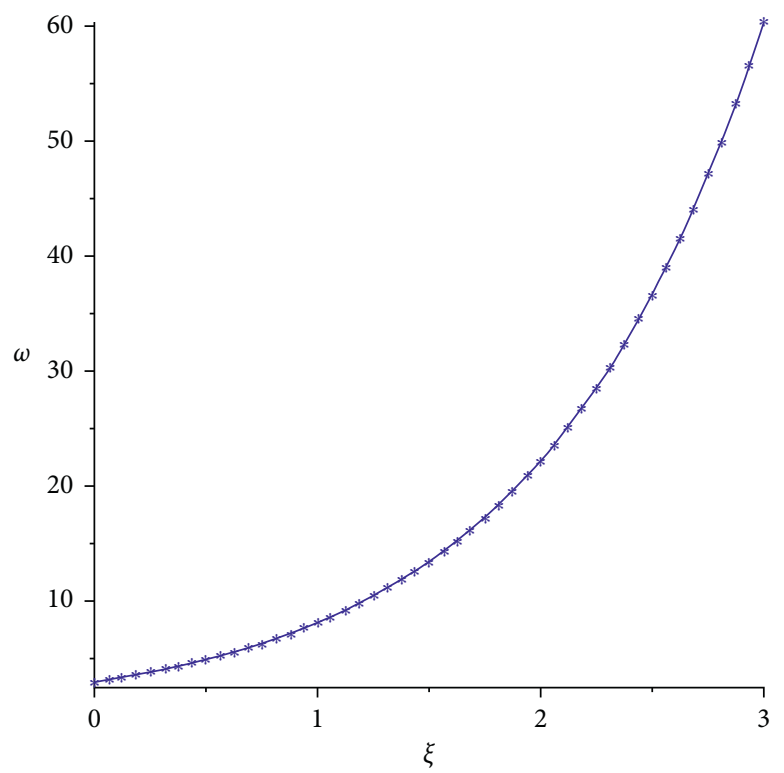

Figure 3: SDM and VITM solution graph of $\omega$ at $\zeta=1$ and $\eta=0.5$ of Example 1.

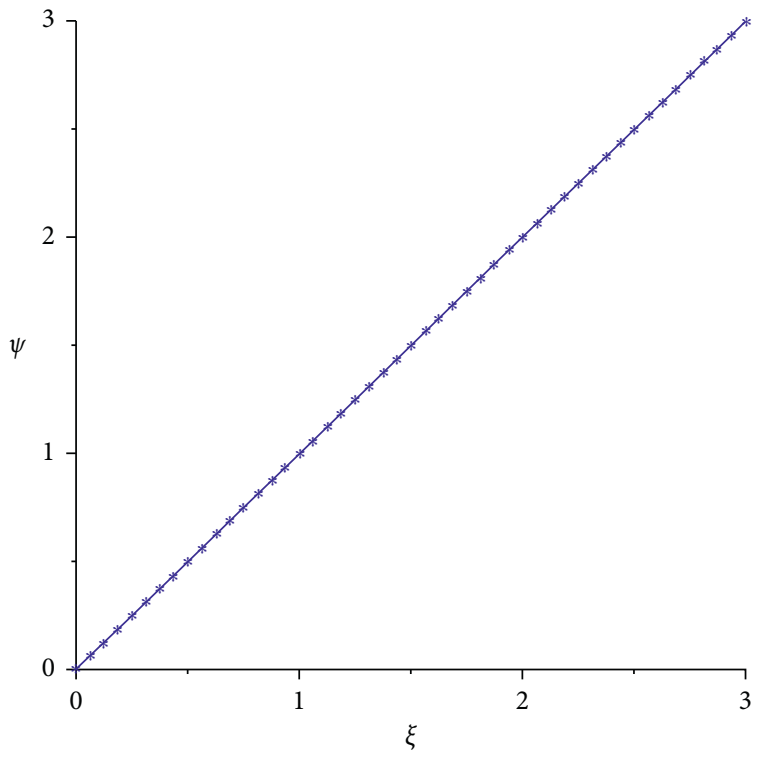

Figure 4: SDM and VITM solution graph of $\psi$ of Example 1.

\section{Results Discussion}

In this section, we discuss the solution-graphs of fractional-order system of nonlinear equations of unsteady flow of a polytropic gas which has been solved by using SDM and VITM. In Figures $1-4$, the solutions $\mu, v, \omega$, and $\psi$ obtained by using SDM and VITM are compared by keeping one variable and other constants. The dotted and line subgraphs are, respectively, denoted the SDM and VITM solutions. It is observed that SDM and VITM solution-graphs are identical and within close

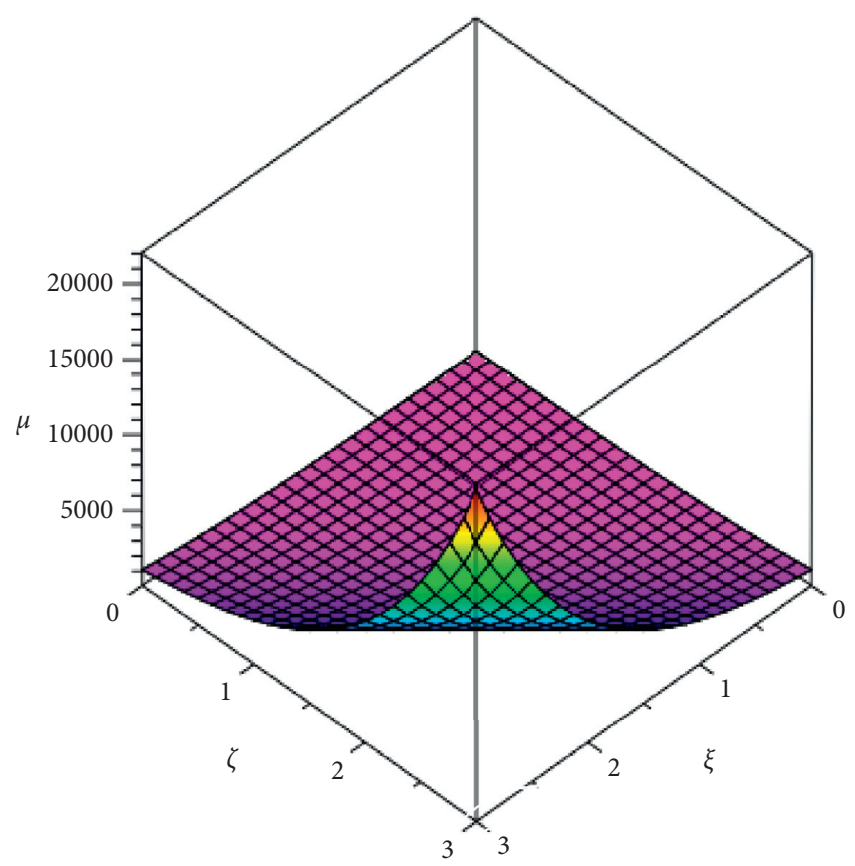

FIgURE 5: SDM and VITM solution 3d graph of $\mu(\xi, \zeta, \eta)$ of Example 1 .

contact. In similar way, in Figures 5-7, the three-dimensional graphs for variables $\mu, \nu$, and $\psi$ are plotted for Example 1. The identical solution-graphs of the suggested methods are attained and confirmed that the results obtained by two different procedures are identical and verified the applicability of the proposed techniques. In Figures $8-10$, the SDM and VITM 


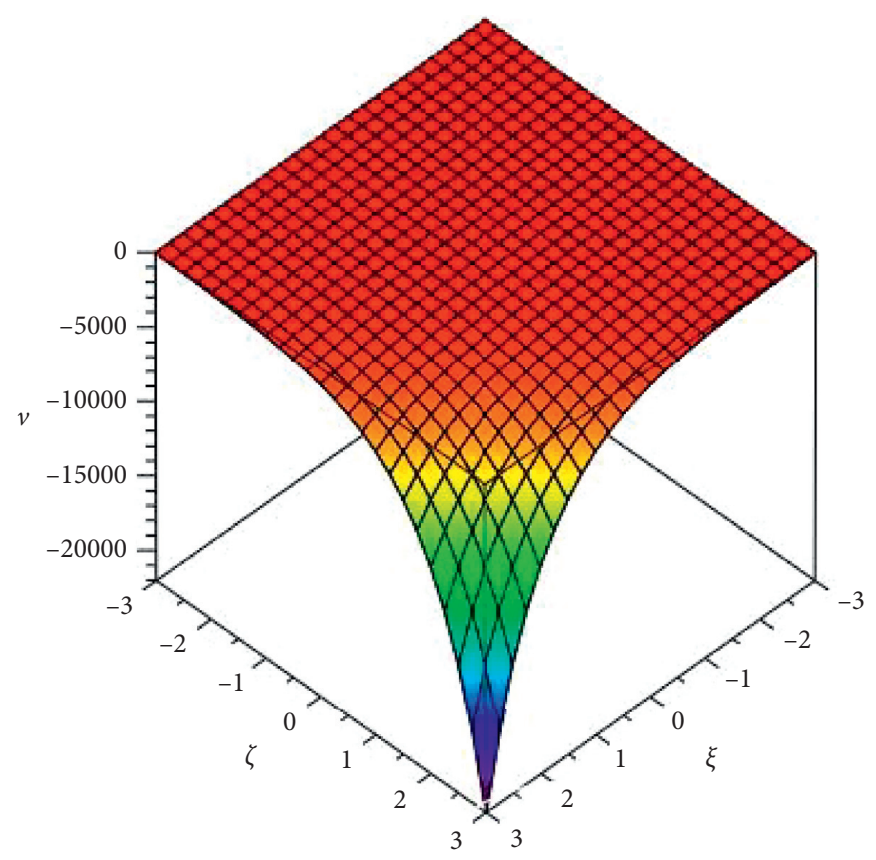

FIGURE 6: SDM and VITM solution 3d graph of $\nu(\xi, \zeta, \eta)$ of Example 1.

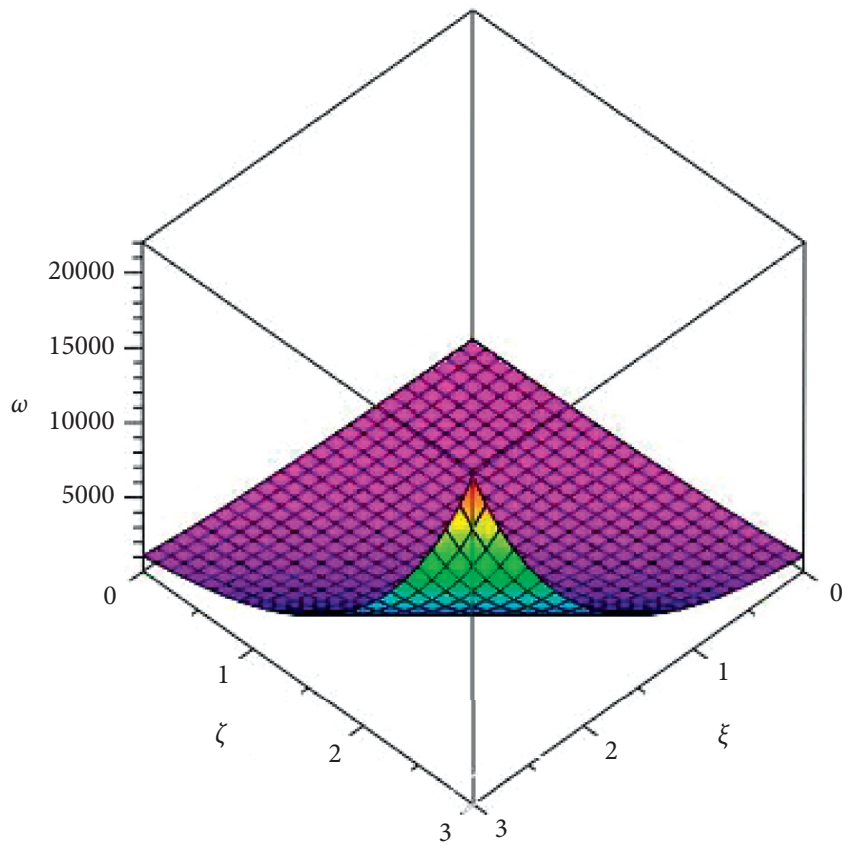

Figure 7: SDM and VITM solution 3d graph of $\omega(\xi, \zeta, \eta)$ of Example 1.

solutions are plotted in two dimensions at fractional order $\delta=$ $0.4,0.6,0.8,1$ for Example 1. The convergence phenomenon of the fractional solutions towards integer solution is observed. The three-dimensional graphs of the fractional-order solutions for Example 1 are represented in Figures 11-13 for variables $\mu, \nu$,

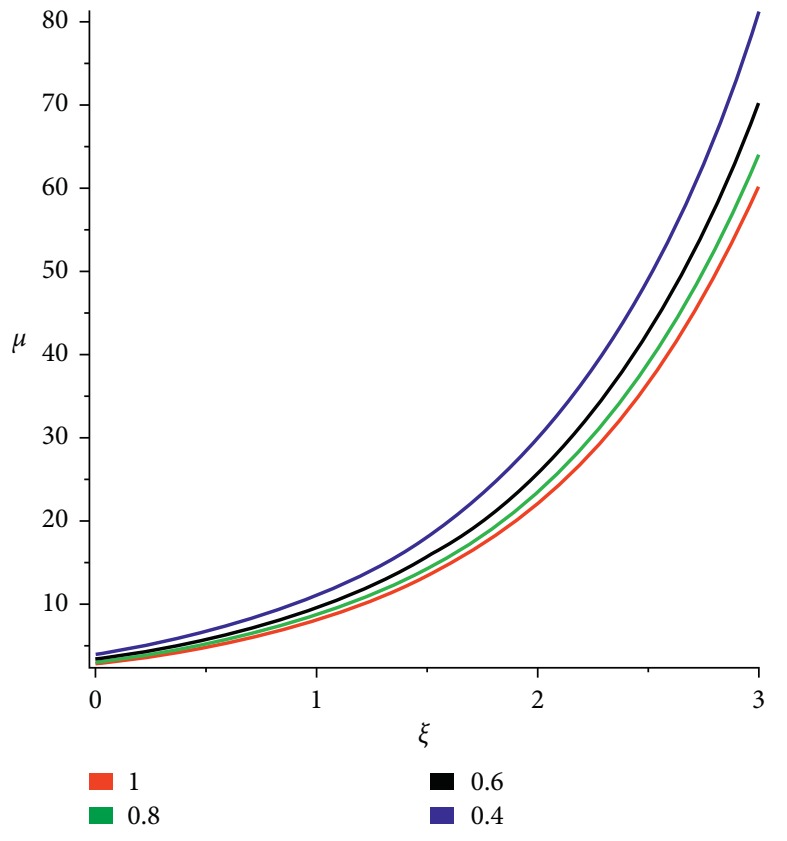

Figure 8: SDM and VITM graph of different value of $\delta$ for $\mu(\xi, \zeta, \eta)$ of Example 1.

and $\omega$, respectively. In Table 1 and Figure 14, the combined graph for variables $\mu, v$, and $\psi$ is displayed at $\delta=1$. The solution comparison of the suggested methods, SDM and VITM, is discussed. The suggested techniques have provided the solutions with the desire degree of accuracy with the consideration of very few terms in its series form solutions. 


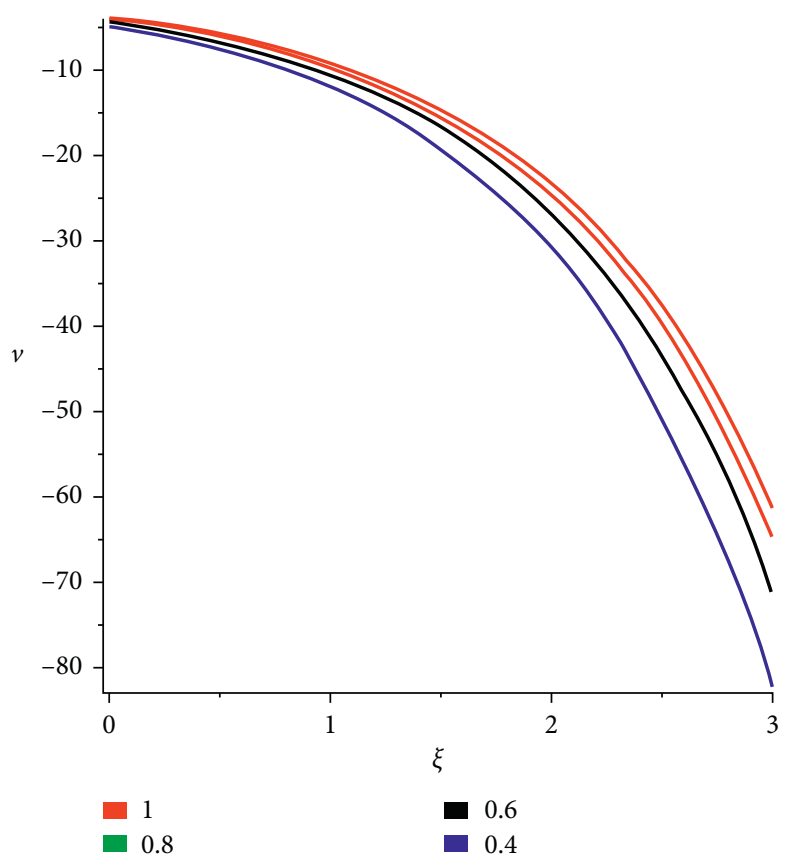

FIgURE 9: SDM and VITM graph of different value of $\delta$ for $\nu(\xi, \zeta, \eta)$ of Example 1.

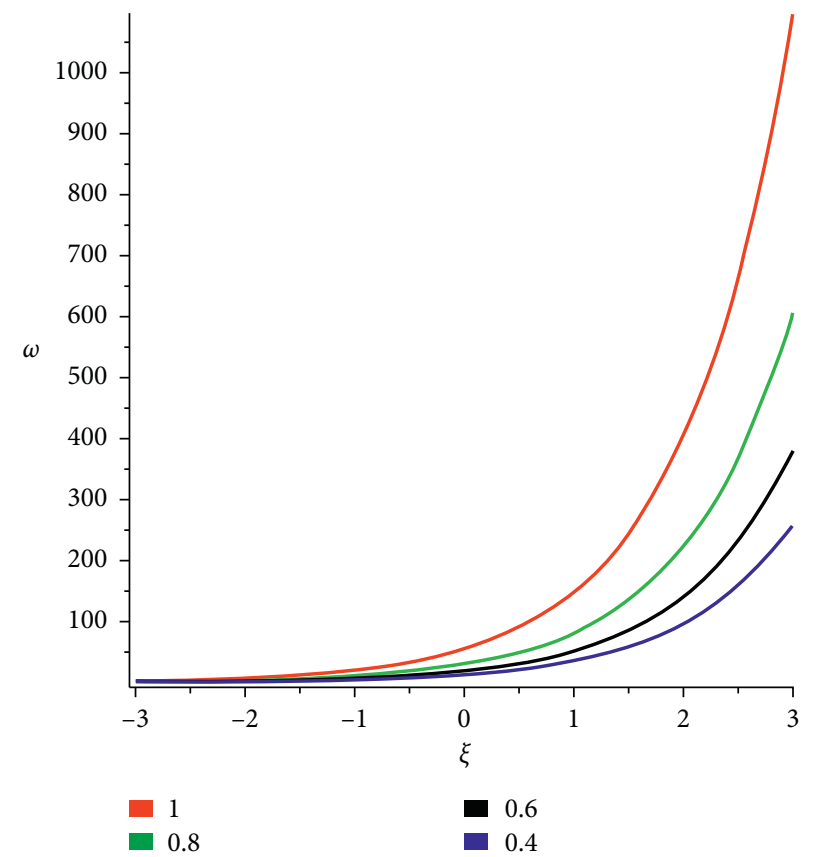

FIgURE 10: SDM and VITM graph of different value of $\delta$ for $\omega(\xi, \zeta, \eta)$ of Example 1. 


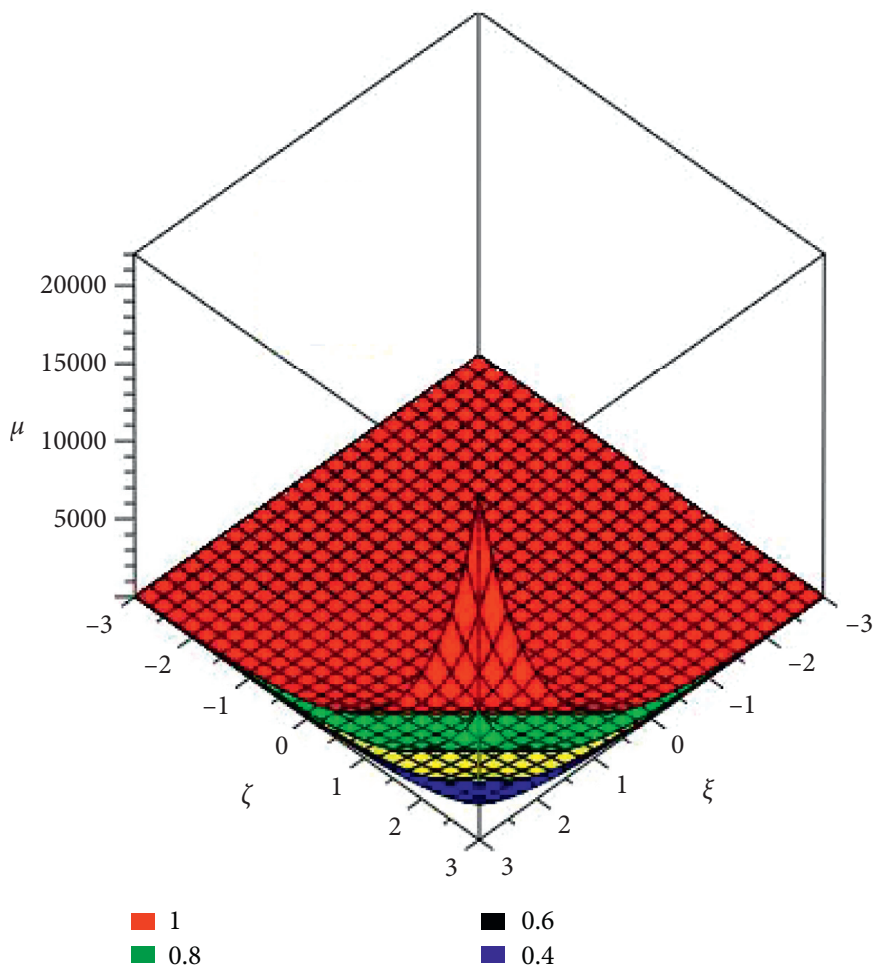

Figure 11: SDM and VITM 3d graph of different value of $\delta$ for $\mu(\xi, \zeta, \eta)$ of Example 1 .

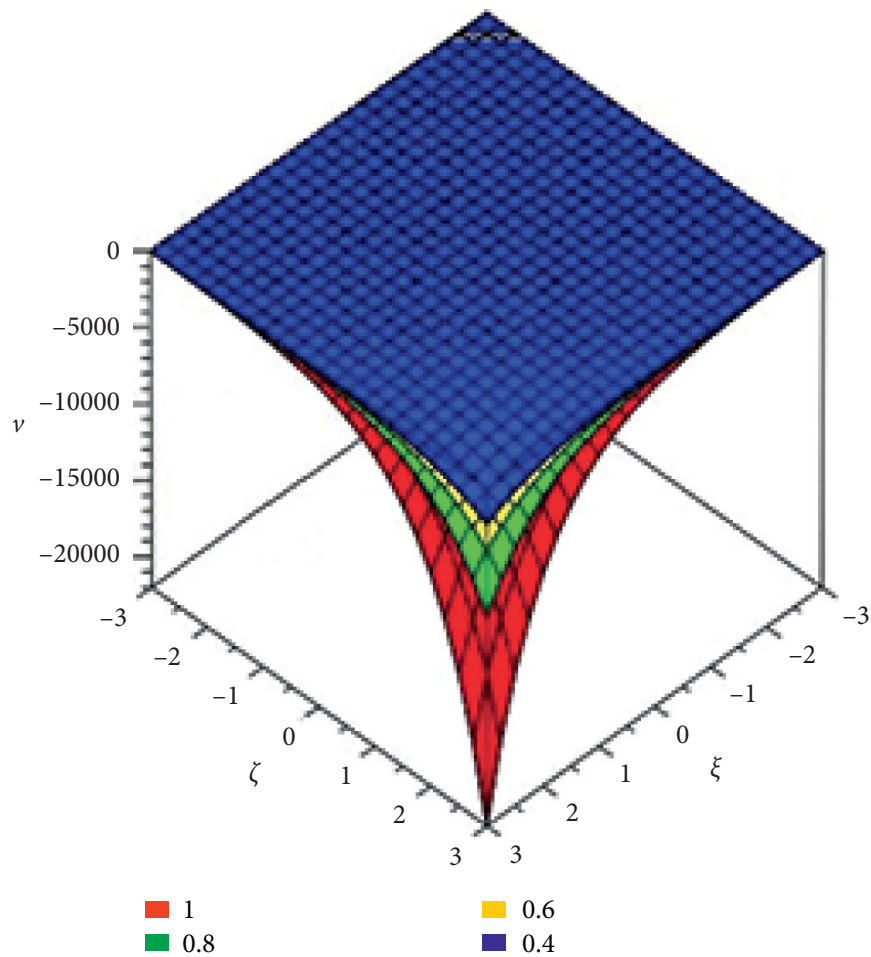

FiguRE 12: SDM and VITM 3d graph of different value of $\delta$ for $\nu(\xi, \zeta, \eta)$ of Example 1. 


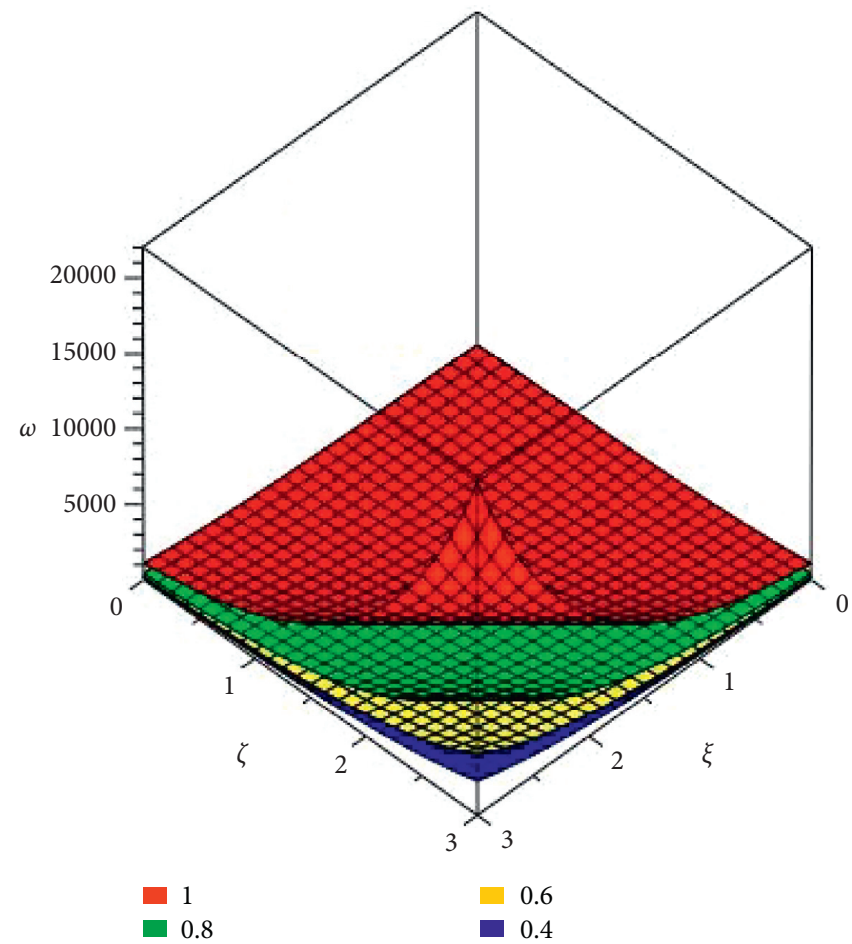

Figure 13: SDM and VITM solution graph of different value of $\delta$ for $\omega(\xi, \zeta, \eta)$ of Example 1 .

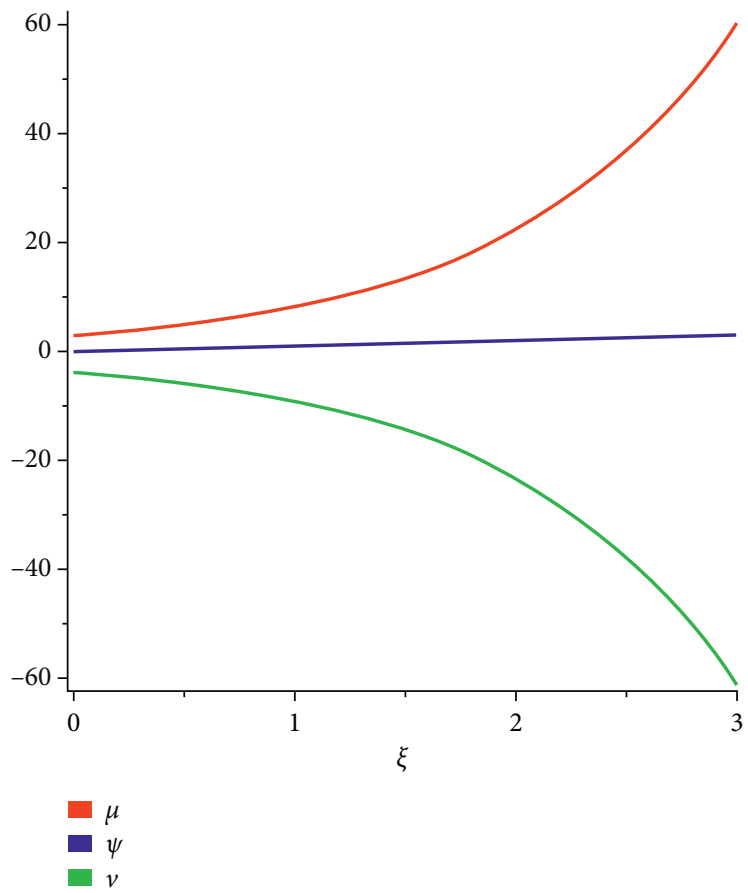

Figure 14: Combine graph of $\mu(\xi, \zeta, \eta), \nu(\xi, \zeta, \eta)$, and $\omega(\xi, \zeta, \eta)$ of Example 1. 
TABLE 1: SDM and VITM solution of Example 1 at $\delta=1, \eta=0.5$, and $\zeta=1$.

\begin{tabular}{lccr}
\hline$\xi$ & $\mathrm{AE} \mathrm{of} \mu$ & $\mathrm{AE} \mathrm{of} \nu$ & $\mathrm{AE}$ of $\omega$ \\
\hline 0.5 & $1.22000 E-07$ & $1.22000 E-07$ & $1.22000 E-07$ \\
1 & $2.01000 E-07$ & $2.01000 E-07$ & $2.01000 E-07$ \\
1.5 & $3.32000 E-07$ & $3.32000 E-07$ & $3.32000 E-07$ \\
2 & $5.50000 E-07$ & $5.50000 E-07$ & $5.50000 E-07$ \\
2.5 & $9.00000 E-07$ & $9.00000 E-07$ & $9.00000 E-07$ \\
3 & $1.49000 E-06$ & $1.49000 E-06$ & $1.49000 E-06$ \\
3.5 & $2.45000 E-06$ & $2.45000 E-06$ & $2.45000 E-06$ \\
4 & $4.06000 E-06$ & $4.06000 E-06$ & $4.06000 E-06$ \\
4.5 & $6.70000 E-06$ & $6.70000 E-06$ & $6.70000 E-06$ \\
5 & $1.11000 E-05$ & $1.11000 E-05$ & $1.11000 E-05$ \\
\hline
\end{tabular}

\section{Conclusion}

In this article, the analytical solution of the system of timefractional partial differential equations of unsteady flow of polytrophic dynamics is investigated by using two different techniques:

(i) The proposed techniques are the mixture of Shehu transformation with Adomian decomposition method and variational iteration method, respectively.

(ii) The obtained solutions of the suggested techniques for both fractional and integer orders are calculated and plotted via two- and three-dimensional graphs.

(iii) A close contact between the actual and the derived results is observed.

(iv) The fractional-order solutions provide various dynamics for a different fractional order of the derivative.

(v) Using analytical solutions, the task can be done rather simple and effective as compared to numerical investigations that need larger calculations.

(vi) After all, the researchers are now able to select the fractional-order problem whose solution is comparatively very close to the experimental results of any physical problem.

(vii) Due to simple and straightforward implementation, the suggested techniques are considered to be preferable to solve other system of FPDEs.

The following abbreviations are used in this article:

\section{Nomenclature}

ST: $\quad$ Shehu transform

LT: $\quad$ Laplace transform

FPDEs: Fractional partial differential equations

VITM: Variational iteration transform method

SDM: Shehu decomposition method

ADM: Adomian decomposition method.

\section{Data Availability}

The data used to support the findings of this study are available from the corresponding author upon request.

\section{Conflicts of Interest}

The authors declare that they have no conflicts of interest.

\section{Authors' Contributions}

H. K. conceptualized the study, prepared the methodology, wrote the original draft, and did formal analysis. M. A. analyzed using the software and supervised the study. S. I. validated, investigated, and visualized the study and administrated the project. H. K. and M. A. managed resources and reviewed and edited the document.

\section{Acknowledgments}

The authors acknowledge Dr. Muhammad Arif, Academic Editor, Mathematical Problems in Engineering, Hindawi.

\section{References}

[1] N. Bertrand, J. Sabatier, O. Briat, and J.-M. Vinassa, “An implementation solution for fractional partial differential equations," Mathematical Problems in Engineering, vol. 2013, Article ID 795651, 7 pages, 2013.

[2] S. S. Ray, A. Atangana, S. C. O. Noutchie, M. Kurulay, N. Bildik, and A. Kilicman, "Fractional calculus and its applications in applied mathematics and other sciences," Mathematical Problems in Engineering, vol. 2014, Article ID 849395, 2 pages, 2014.

[3] B. Zheng, "Exact solutions for some fractional partial differential equations by the method," Mathematical Problems in Engineering, vol. 2013, Article ID 826369, 13 pages, 2013.

[4] P. Goswami and R. T. Alqahtani, "On the solution of local fractional differential equations using local fractional Laplace variational iteration method," Mathematical Problems in Engineering, vol. 2016, Article ID 9672314, 6 pages, 2016.

[5] R. Shah, H. Khan, P. Kumam, and M. Arif, "An analytical technique to solve the system of nonlinear fractional partial differential equations," Mathematics, vol. 7, no. 6, p. 505, 2019.

[6] J. Singh, "Analysis of fractional blood alcohol model with composite fractional derivative," Chaos, Solitons \& Fractals, vol. 140, Article ID 110127, 2020.

[7] D. Kumar, J. Singh, and D. Baleanu, "On the analysis of vibration equation involving a fractional derivative with Mittag-Leffler law," Mathematical Methods in the Applied Sciences, vol. 43, no. 1, pp. 443-457, 2020.

[8] S. Bhatter, A. Mathur, D. Kumar, K. S. Nisar, and J. Singh, "Fractional modified Kawahara equation with Mittag-Leffler 
law," Chaos, Solitons \& Fractals, vol. 131, Article ID 109508, 2020.

[9] K. K. Ali, M. S. Osman, H. M. Baskonus, N. S. Elazabb, and E. Ilhan, "Analytical and numerical study of the HIV-1 infection of CD4+ T-cells conformable fractional mathematical model that causes acquired immunodeficiency syndrome with the effect of antiviral drug therapy," Mathematical Methods in the Applied Sciences, 2020.

[10] Y. Zhang, C. Cattani, and X. J. Yang, "Local fractional homotopy perturbation method for solving non-homogeneous heat conduction equations in fractal domains," Entropy, vol. 17, no. 10, pp. 6753-6764, 2015.

[11] W. Gao, H. Günerhan, and H. M. Baskonus, "Analytical and approximate solutions of an epidemic system of HIV/AIDS transmission," Alexandria Engineering Journal, vol. 59, no. 5, pp. 3197-3211, 2020.

[12] W. Gao, P. Veeresha, D. G. Prakasha, and H. M. Baskonus, "New numerical simulation for fractional Benney-Lin equation arising in falling film problems using two novel techniques," Numerical Methods for Partial Differential Equations, vol. 37, no. 1, pp. 210-243, 2020.

[13] J. Singh, D. Kumar, Z. Hammouch, and A. Atangana, "A fractional epidemiological model for computer viruses pertaining to a new fractional derivative," Applied Mathematics and Computation, vol. 316, pp. 504-515, 2018.

[14] W. Gao, H. M. Baskonus, and L. Shi, "New investigation of bats-hosts-reservoir-people coronavirus model and application to 2019-nCoV system," Advances in Difference Equations, vol. 2020, no. 1, pp. 1-11, 2020.

[15] D. Kumar, J. Singh, K. Tanwar, and D. Baleanu, "A new fractional exothermic reactions model having constant heat source in porous media with power, exponential and MittagLeffler laws," International Journal of Heat and Mass Transfer, vol. 138, pp. 1222-1227, 2019.

[16] M. Sheikholeslami, M. Nimafar, and D. D. Ganji, "Nanofluid heat transfer between two pipes considering Brownian motion using AGM," Alexandria Engineering Journal, vol. 56, no. 2, pp. 277-283, 2017.

[17] G. D. Domari, M. Peiravi, and M. Abbasi, "Evaluation of the heat transfer rate increases in retention pools nuclear waste," International Journal of Nano Dimension, vol. 6, no. 4, 2015.

[18] M. M. Peiravi, J. Alinejad, D. Ganji, and S. Maddah, "Numerical study of fins arrangement and nanofluids effects on three-dimensional natural convection in the cubical enclosure," Transport Phenomena in Nano and Micro Scales, vol. 7, no. 2, pp. 97-112, 2019.

[19] M. M. Peiravi and J. Alinejad, "Hybrid conduction, convection and radiation heat transfer simulation in a channel with rectangular cylinder," Journal of Thermal Analysis and Calorimetry, vol. 140, pp. 1-15, 2019.

[20] J. Alinejad and M. M. Peiravi, "Numerical analysis of secondary droplets characteristics due to drop impacting on 3D cylinders considering dynamic contact angle," Meccanica, vol. 55, no. 10, pp. 1975-2002, 2020.

[21] M. M. Peiravi, J. Alinejad, D. D. Ganji, and S. Maddah, “3D optimization of baffle arrangement in a multi-phase nanofluid natural convection based on numerical simulation," International Journal of Numerical Methods for Heat \& Fluid Flow, vol. 30, no. 5, 2019.

[22] M. Yavuz and N. Özdemir, "European vanilla option pricing model of fractional order without singular kernel," Fractal and Fractional, vol. 2, no. 1, p. 3, 2018.

[23] M. Yavuz and N. Özdemir, "Numerical inverse Laplace homotopy technique for fractional heat equations," 2018.
[24] M. Yavuz and N. Özdemir, "A quantitative approach to fractional option pricing problems with decomposition series," Konuralp Journal of Mathematics, vol. 6, no. 1, pp. 102-109, 2018.

[25] M. Yavuz, "Novel solution methods for initial boundary value problems of fractional order with conformable differentiation," An International Journal of Optimization and Control: Theories \& Applications (IJOCTA), vol. 8, no. 1, pp. 1-7, 2017.

[26] M. Yavuz and T. Abdeljawad, "Nonlinear regularized longwave models with a new integral transformation applied to the fractional derivative with power and Mittag-Leffler Kernel," Advances in Difference Equations, vol. 367, no. 1, pp. 1-18, 2020.

[27] M. Yavuz and N. Özdemir, "A different approach to the European option pricing model with new fractional operator," Mathematical Modelling of Natural Phenomena, vol. 13, no. 1, p. 12, 2018.

[28] F. Evirgen, "Conformable fractional gradient based dynamic system for constrained optimization problem," Acta Physica Polonica A, vol. 132, no. 3, pp. 1066-1069, 2017.

[29] N. Özdemir, S. Uçar, and B. B. I. Eroglu, "Dynamical analysis of fractional order model for computer virus propagation with kill signals," International Journal of Nonlinear Sciences and Numerical Simulation, vol. 21, no. 3-4, 2019.

[30] A. Goswami, J. Singh, and D. Kumar, "An efficient analytical approach for fractional equal width equations describing hydro-magnetic waves in cold plasma," Physica A: Statistical Mechanics and Its Applications, vol. 524, pp. 563-575, 2019.

[31] K. R. Raslan, K. K. Ali, and M. A. Shallal, "The modified extended tanh method with the Riccati equation for solving the space-time fractional EW and MEW equations," Chaos, Solitons \& Fractals, vol. 103, pp. 404-409, 2017.

[32] H. Rezazadeh, A. Korkmaz, M. M. A. Khater, M. Eslami, D. Lu, and R. A. M. Attia, "New exact traveling wave solutions of biological population model via the extended rational sinh-cosh method and the modified Khater method," Modern Physics Letters B, vol. 33, no. 28, Article ID 1950338, 2019.

[33] A. H. Salas, S. A. El-Tantawy, and H. Castillo, "The hybrid finite difference and moving boundary methods for solving a linear damped nonlinear schrödinger equation to model rogue waves and breathers in plasma physics," Mathematical Problems in Engineering, vol. 2020, p. 11, Article ID 6874870, 2020.

[34] Y. Qin, A. Khan, I. Ali et al., "An efficient analytical approach for the solution of certain fractional-order dynamical systems," Energies, vol. 13, no. 11, p. 2725, 2020.

[35] R. Shah, H. Khan, D. Baleanu, P. Kumam, and M. Arif, "A novel method for the analytical solution of fractional Zakharov-Kuznetsov equations," Advances in Difference Equations, vol. 2019, no. 1, pp. 1-14, 2019.

[36] P. Veeresha, D. G. Prakasha, and H. M. Baskonus, "An efficient technique for a fractional-order system of equations describing the unsteady flow of a polytropic gas," Pramana, vol. 93 , no. 5, p. 75, 2019.

[37] H. Moradpour, A. Abri, and H. Ebadi, "Thermodynamic behavior and stability of Polytropic gas," International Journal of Modern Physics D, vol. 25, no. 1, p. 1650014, 2016.

[38] M. Matinfar and M. Saeidy, "Homotopy analysis method for solving the equation governing the unsteady flow of a polytropic gas," World Applied Sciences Journal, vol. 9, no. 9, pp. 980-983, 2010.

[39] M. Tamsir and V. K. Srivastava, "Revisiting the approximate analytical solution of fractional-order gas dynamics 
equation," Alexandria Engineering Journal, vol. 55, no. 2, pp. 867-874, 2016.

[40] P. K. G. Bhadane and V. H. Pradhan, "Elzaki transform homotopy perturbation method for solving gas dynamics equation," International Journal of Research in Engineering and Technology, vol. 2, no. 12, pp. 260-264, 2013.

[41] M. A. Mohamed, "Adomian decomposition method for solving the equation governing the unsteady flow of a polytropic gas," Applied Mathematics, vol. 4, no. 1, pp. 52-61, 2009.

[42] M. H. Cherif, D. Ziane, and K. Belghaba, "Fractional natural decomposition method for solving fractional system of nonlinear equations of unsteady flow of a polytropic gas," Nonlinear Studies, vol. 25, pp. 753-764, 2018.

[43] N. Anjum and J.-H. He, "Laplace transform: making the variational iteration method easier," Applied Mathematics Letters, vol. 92, pp. 134-138, 2019.

[44] M. O. Olayiwola, "Solutions of emden-fowler type equations by variational iteration method," Çankaya Üniversitesi Bilim Ve Mühendislik Dergisi, vol. 16, no. 2, pp. 1-9, 2019.

[45] M. Nadeem, F. Li, and H. Ahmad, "Modified Laplace variational iteration method for solving fourth-order parabolic partial differential equation with variable coefficients," Computers \& Mathematics with Applications, vol. 78, no. 6, pp. 2052-2062, 2019.

[46] J.-H. He, "Variational iteration method - a kind of non-linear analytical technique: some examples," International Journal of Non-linear Mechanics, vol. 34, no. 4, pp. 699-708, 1999.

[47] J. H. He and X. H. Wu, "Variational iteration method: new development and applications," Computers \& Mathematics with Applications, vol. 54, no. 7-8, pp. 881-894, 2007.

[48] G.-c. Wu and E. W. M. Lee, "Fractional variational iteration method and its application," Physics Letters A, vol. 374, no. 25, pp. 2506-2509, 2010.

[49] R. Shah, H. Khan, D. Baleanu, P. Kumam, and M. Arif, "A semi-analytical method to solve family of Kuramoto-Sivashinsky equations," Journal of Taibah University for Science, vol. 14, no. 1, pp. 402-411, 2020.

[50] A.-M. Wazwaz, "A reliable modification of Adomian decomposition method," Applied Mathematics and Computation, vol. 102, no. 1, pp. 77-86, 1999.

[51] S. Maitama and W. Zhao, "New integral transform: Shehu transform a generalization of Sumudu and Laplace transform for solving differential equations," 2019, http://arxiv.org/abs/ 1904.11370.

[52] H. Khan, U. Farooq, R. Shah, D. Baleanu, P. Kumam, and M. Arif, "Analytical solutions of (2+ time fractional order) dimensional physical models, using modified decomposition method," Applied Sciences, vol. 10, no. 1, p. 122, 2020.

[53] A. Khalouta and A. Kadem, "A comparative study of Shehu variational iteration method and Shehu decomposition method for solving nonlinear Caputo time-fractional wavelike equations with variable coefficients," Applications \& Applied Mathematics, vol. 15, no. 1, 2020.

[54] L. Akinyemi and O. S. Iyiola, "Exact and approximate solutions of time-fractional models arising from physics via Shehu transform," Mathematical Methods in the Applied Sciences, 2020.

[55] J. A. T. Machado, D. Baleanu, W. Chen, and J. Sabatier, "New trends in fractional dynamics," Journal of Vibration and Control, vol. 20, no. 7, p. 963, 2014.

[56] D. Baleanu, Z. Guvenc, and J. Machado, New Trends in Nanotechnology and Fractional Calculus Applications, Springer, Dordrecht, Netherlands, 2010.
[57] A. Bokhari, D. Baleanu, and R. Belgacem, "Application of Shehu transform to atangana-baleanu derivatives," Journal of Mathematics and Computer Science, vol. 20, no. 2, pp. 101107, 2019.

[58] R. Belgacem, D. Baleanu, and A. Bokhari, "Shehu transform and applications to caputo-fractional differential equations," International Journal of Analysis and Applications, vol. 17, pp. 917-927, 2019.

[59] Z. M. Odibat, "A study on the convergence of variational iteration method," Mathematical and Computer Modelling, vol. 51, no. 9-10, pp. 1181-1192, 2010.

[60] H. A. Zedan, S. S. Tantawy, and Y. M. Sayed, "Convergence of the variational iteration method for initial-boundary value problem of fractional integro-differential equations," Journal of Fractional Calculus and Applications, vol. 5, no. 3, pp. 1-14, 2014. 